\title{
Cross-diffusion and pattern formation in reaction-diffusion systems
}

\author{
Vladimir K. Vanag*† and Irving R. Epstein* \\ Received 12th August 2008, Accepted 2nd October 2008 \\ First published as an Advance Article on the web 11th December 2008 \\ DOI: $10.1039 / b 813825 g$
}

Cross-diffusion, the phenomenon in which a gradient in the concentration of one species induces a flux of another chemical species, has generally been neglected in the study of reaction-diffusion systems. We summarize experiments that demonstrate that cross-diffusion coefficients can be quite significant, even exceeding "normal," diagonal diffusion coefficients in magnitude in systems that involve ions, micelles, complex formation, excluded volume effects (e.g., surface or polymer reactions) and other phenomena commonly encountered in situations of interest to chemists. We then demonstrate with a series of model calculations that cross-diffusion can lead to spatial and spatiotemporal pattern formation, even in relatively simple systems. We also show that, in the absence of cross-diffusion among the reacting species, introduction of a nonreactive species that induces appropriate cross-diffusive fluxes with reactive species can lead to pattern formation.

\section{Introduction}

Reaction-diffusion (RD) systems have attracted increasing attention from the scientific community in recent years as investigators have begun to seek insights into the fascinating patterns that occur in living organisms, in ecological systems, in geochemistry and in physicochemical systems. The rapid growth of the field of systems biology has further contributed to interest in RD systems. Thermodynamically stable spatial structures, ${ }^{1}$ like crystals (for example, snowflakes ${ }^{2}$ ), micelles, membranes and other aggregates are said to arise via selfassembly. Another class of structures, of which Turing patterns ${ }^{3}$ are perhaps the best known example, occur only far from equilibrium. They are said to involve self-organization and

Department of Chemistry and Volen Center for Complex Systems, MS015, Brandeis University, 415 South St., Waltham, MA 02454, USA.E-mail:vanag@brandeis.edu; epstein@brandeis.edu

$\dagger$ Also, Department of Biology, Lomonosov Moscow State University, Moscow 119899, Russia. result from mechanisms that may be called kinetic or dissipative. Introduced theoretically by Turing, ${ }^{3}$ extensively studied by Prigogine and collaborators, ${ }^{4}$ and then realized experimentally in Bordeaux, ${ }^{5}$ Austin, ${ }^{6}$ and Brandeis ${ }^{7}$ among other places, these routes to pattern formation couple nonlinear chemical kinetics and diffusion. These dissipative or nonequilibrium patterns include spiral waves, ${ }^{8}$ Turing patterns, ${ }^{5,7}$ standing waves ${ }^{7,9}$ and other spatiotemporal phenomena that require an input of energy and/or matter.

Diffusion processes play a role in both self-assembly and selforganization, while chemical kinetics is important only in the latter case. Detailed understanding of self-assembly requires knowledge of the explicit form of the interaction potential between particles or molecules, which allows us to calculate such thermodynamic functions as energy and entropy. In chemical kinetics, the mechanisms of chemical and physical interaction between species are hidden in the reaction rate constants, which significantly simplifies the description of chemical processes. When analyzing $\mathrm{RD}$ mechanisms, one

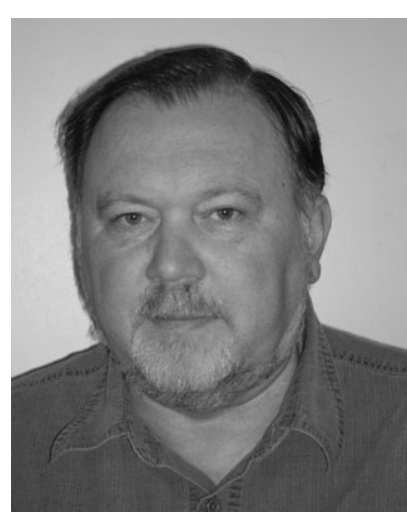

Vladimir Karl Vanag
Vladimir Karl Vanag, biophysicist, graduated from Moscow State University (MSU) in 1977. He obtained his first scientific degree, $P h D$, in 1985 and the second, Doctor of Sciences, in 1996. His craving for nonlinear science started in the late $80 \mathrm{~s}$ at the Institute of Chemical Physics of the Russian Academy of Sciences, where he served as a Leading Scientist. He worked as Visiting Professor at the Institute for Molecular Science (Japan, Okazaki, 1993-1994). He received a governmental medal from the President of the Russian Federation. In 1998, he moved to Brandeis University. He also lectures on "Pattern formation" at MSU.

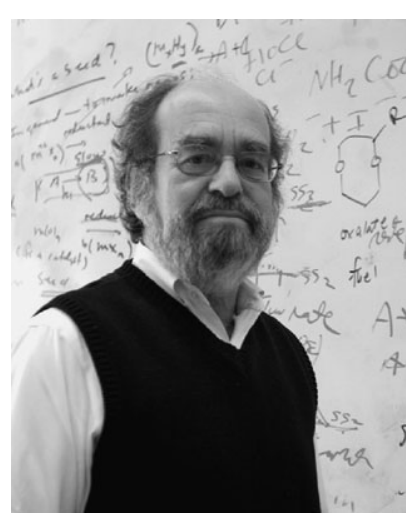

Irving R. Epstein
Irving R. Epstein is Henry F. Fischbach Professor of Chemistry, Howard Hughes Medical Institute Professor and chair of the Chemistry Department at Brandeis University. $\mathrm{He}$ received his $A B, M A$ and $P h D$ degrees from Harvard University and a Diploma in Advanced Mathematics as a Marshall Scholar at Oxford University. He chaired the first Gordon Research Conference on Chemical Oscillations and Dynamic Instabilities and currently serves as Associate Editor of Chaos and chair of the board of directors of the New England Complex Systems Institute. 
usually assumes that chemical and diffusion processes can be separated without any harm, at least for relatively slow reactions. Note, however, that there is a well known and venerable example in which a diffusion process directly determines the rate of a chemical process, namely a diffusion-controlled bimolecular reaction, whose rate, $k_{\mathrm{d}}[\mathrm{A}][\mathrm{B}]$, is characterized by $^{10-13}$

$$
k_{\mathrm{d}}=4 \pi f \rho D N_{\mathrm{A}}
$$

where $k_{\mathrm{d}}$ is the diffusion-controlled reaction rate constant, $\rho$ is the encounter diameter of the molecules $\mathrm{A}$ and $\mathrm{B}, D=D_{\mathrm{A}}+D_{\mathrm{B}}$ is the diffusion coefficient of their relative motion and $N_{\mathrm{A}}$ is Avogadro's number. The factor $f$ takes into account the charges on species A and B. If their charges have the same (opposite) signs, then $f<(>) 1$, and $f \cong 1$ for neutral A or $\mathrm{B}$.

Nearly all efforts to explain the patterns found experimentally in RD systems, such as the CIMA (chlorite-iodide-malonic acid) reaction, ${ }^{5,6}$ the BZ-AOT (Belousov-Zhabotinsky in aerosol OT microemulsion) system ${ }^{7,14}$ or the FIS (ferrocyanide-iodide-sulfite) reaction, ${ }^{15-17}$ employ only the diagonal elements of the diffusion matrix. That is, they assume that the diffusion of species A depends only upon gradients in the concentration of A, and not on the spatial distribution of any other species. In this case, an RD system can be written in the following general form

$$
\partial u_{i} / \partial t=R_{i}(\mathbf{u})+D_{i i} \nabla^{2} u, i=1,2, \ldots, N
$$

where the vector $\mathbf{u}$ consists of the concentrations $u_{j}(j=1,2, \ldots, N)$ of the $N$ species, the $R_{i}(\mathbf{u})$ give the reaction kinetics, and $D_{i i}$ is the diffusion coefficient of the $i$ th species. Patterns found in such RD systems generally arise via either Turing instability or wave instability. ${ }^{3}$ These instabilities can lead to patterns in spatially extended systems if the homogeneous steady state is subjected to an infinitesimal perturbation. ${ }^{5,7}$ In some cases, patterns can emerge only if a sufficiently large perturbation with a suitable spatial structure is applied. ${ }^{18-20}$ This latter (subcritical) group of mechanisms can also produce localized patterns. $^{21}$

The Turing instability, which leads to patterns that are stationary in time and periodic in space, can be characterized as arising from "long range inhibition and short range activation", at least for two-variable models. Translating these biologicallyoriented terms into physicochemical ones, we may say that the autocatalytic species (activator) must have a diffusion coefficient smaller (often much smaller) than that of the other (inhibitor) species. The conditions for wave instability, which can occur only in systems with three or more variables, and which leads to patterns periodic in both time and space, are more complex. Typically, a third species coupled to the activator must have a diffusion coefficient that differs significantly from the diffusion coefficients of both the activator and the inhibitor.

Chemical and physical interaction between species can be concealed not only in the reaction rate constants, as mentioned above, but also in the parameters used to describe the diffusion process, especially when cross-diffusion is present. Cross-diffusion refers to the phenomenon in which a flux of species $\mathrm{A}$ is induced by a gradient of species B. Such processes can be characterized by the Fickian diffusivity matrix $\mathbf{D}$ or by the Maxwell-Stefan diffusion matrix $\mathscr{D}$, where the diffusion coefficient $\mathscr{D}_{i j}$ has the physical meaning of an inverse drag coefficient in the Maxwell-Stefan equations, ${ }^{22}$ rather than by a vector of coefficients $D_{i i}$. Examples of systems that exhibit cross-diffusion include strong electrolytes, ${ }^{23-25}$ micelles, ${ }^{26,27}$ or microemulsions, ${ }^{28}$ and systems containing molecules of significantly different sizes, for example protein-salt. ${ }^{29-31}$ Similar phenomena also occur in biological systems, but since the transport process is driven by an input of energy, as, for example, in bacterial chemotaxis ${ }^{32-34}$ or in predator-prey systems, ${ }^{35-38}$ these are not true diffusion processes. Nonetheless, the mathematical description of biological or ecological cross-diffusion is the same as in physicochemical systems, as we discuss below.

Following the Fickian interpretation of the diffusion process, the general RD equations with cross-diffusion terms take the form:

$$
\begin{aligned}
\partial u_{i} / \partial t= & R_{i}(\mathbf{u})+D_{i i} \nabla^{2} u_{i}+\sum_{j \neq i} \operatorname{div}\left(D_{i j} \nabla u_{j}\right), \\
i, j=1,2, \ldots, N &
\end{aligned}
$$

where the new term, $\Sigma \operatorname{div}\left(D_{i j} \nabla u_{j}\right)$, takes into account the flux of $u_{i}, D_{i j} \nabla u_{j}$, induced by the gradient of species $u_{j}$. Note that eqn (3) can include nonreactive species for which the reactive term $R_{i}(\mathbf{u})$ equals zero. These species may be crucial for pattern formation, since cross-diffusion between reactive and nonreactive species can induce significant fluxes of reactive species.

In this Perspective, we first describe several processes that can give rise to cross-diffusion, like chemotaxis, ${ }^{39}$ weak nonbonding solute-solute interactions, ${ }^{40,41}$ electrostatic ${ }^{42,43}$ or excluded volume effects. ${ }^{30,42,44-49}$ Methods of measurement of crossdiffusion constants are briefly described. We present our own theoretical results that predict patterns in very simple chemical systems (described by eqn (3)), patterns that do not require the usual condition of activator-inhibitor species having significantly different diffusion coefficients.

\section{Cross-diffusion without reaction}

At first glance, diffusion might seem to be a simple process, but in multi-component systems this is rarely the case. The complex range of possibilities becomes apparent if we simply consider the various terms used to characterize diffusive processes. Indeed, such expressions as self-diffusion, tracer diffusion, intradiffusion and interdiffusion, mutual diffusion, cross-diffusion, uphill diffusion $^{50,51}$ and incongruent or negative diffusion ${ }^{52,53}$ require careful definition. If we look back at the history of diffusion, we find that its experimental investigation in binary liquid mixtures began in $1850,{ }^{54}$ and its theoretical description started in 1855 with Fick. ${ }^{55}$ The possibility of cross-terms in multi-component systems was suggested only in $1932 .{ }^{56}$ In 1955, Gosting and collaborators undertook the experimental verification of the existence of cross-diffusion. ${ }^{57,58}$ They used optical interferometry, Gouy and Rayleigh methods. ${ }^{59,60}$ Other approaches, like the diaphragm-cell technique ${ }^{61-63}$ and the Taylor method ${ }^{64-67}$ have also been adapted to measure cross-diffusion coefficients. The Taylor method, which we discuss below, is probably the most widely employed technique at present.

The term "mutual diffusion coefficients" is used as a synonym for interdiffusion coefficients. These are the coefficients used in eqn (3), both the diagonal and off-diagonal elements of 
matrix D. For a binary solution, only a single coefficient is necessary to describe diffusion, while for a ternary solution, we already need four diffusion coefficients, and nine are required for quaternary systems. These diffusion coefficients, including the diagonal elements of the matrix $\mathbf{D}$. are dependent on both concentration and temperature. Negative diffusion provides a striking example of the dependence of $D_{i i}$ on concentration. ${ }^{52,53}$

The term intradiffusion was probably first introduced by Albright and Mills. ${ }^{68,69}$ Following these authors, in the book by Tyrrell and Harris, ${ }^{70}$ self-diffusion is considered as a special case of intradiffusion in a system which contains only two distinguishable (by isotopic labeling, for example) forms of the same chemical species. Self-diffusion can be measured at low concentrations when the interaction between particles is negligible. As concentrations approach zero, the diagonal elements of $\mathbf{D}$ tend to the self-diffusion coefficients.

The self-diffusion coefficient of any component $\alpha$ in a system can be defined as ${ }^{71}$

$$
D_{\alpha}=\frac{1}{3 N_{\alpha}} \sum_{i=1}^{N_{\alpha}} \int_{0}^{\infty}\left\langle\vec{u}_{i}(t) \vec{u}_{i}\right\rangle \mathrm{d} t
$$

where $\left\langle\vec{u}_{i}(t) \vec{u}_{i}\right\rangle$ is the velocity autocorrelation function of an individual particle and $N_{\alpha}$ is the number of these particles. ${ }^{72}$ From this definition, it was noted ${ }^{73}$ that, unlike mutual diffusion coefficients, self-diffusion coefficients are independent of reference frame and of the chemical potential derivatives. Relations between self-diffusion and mutual (interdiffusion) diffusion coefficients can be established, ${ }^{73,74}$ for example, as Darken's equation ${ }^{75,76}$ or the Hartley-Crank relation. ${ }^{77,78}$

Since diffusion coefficients characterize the motion of particles, and the velocity of any motion is measured in some reference frame, the choice of reference frame is very important for determining diffusion coefficients. ${ }^{53}$ The diffusion flows (or fluxes) in some arbitrary reference frame $\mathrm{R}$ can be written as

$$
J_{j}^{\mathrm{R}}=c_{j}\left(u_{j}-u_{\mathrm{R}}\right)
$$

where $c_{j}$ is the concentration of component $j$, and $u_{j}$ and $u_{\mathrm{R}}$ are the local velocities of $j$ and the reference frame $\mathrm{R}$, respectively. Any one component flow (for $n$ components) can be eliminated by the definition of the reference frame via the relation

$$
\sum_{j=1}^{n} a_{j}^{\mathrm{R}} J_{j}^{\mathrm{R}}=0
$$

where $a_{j}^{\mathrm{R}}$ is an appropriate weighting factor. Usually, it is the solvent that is eliminated. The final form of Fick's equations (analogous to eqn (3) without reaction terms) contains diffusion coefficients $D_{i j}$ that depend on the reference frame.

There are three important reference frames, the solvent-fixed reference frame, the mass-fixed (or barycentric) reference frame, and the volume-fixed reference frame. For these frames, the coefficients $a_{j}^{\mathrm{R}}$ are as follows: all $a_{j}^{0}=0$ except $a_{n}^{0}=1$ for the solvent-fixed frame, where $n$ corresponds to solvent molecules and the superscript 0 denotes the solvent-fixed frame; $a_{j}^{\mathrm{M}}=M_{j}$ for the mass-fixed frame, where $M_{j}$ is the molecular mass; and $a_{i}^{\mathrm{V}}=\bar{V}_{i}$ for the volume fixed frame, where $\bar{V}_{i}$ is the partial molar volume of component $i$. For most laboratory diffusion experiments, the volume-fixed reference frame is most appropriate.
There are well-established rules for transformations of diffusion coefficients from one reference frame to another. ${ }^{53,79}$

Independent of the reference frame, the matrix of diffusion coefficients must obey constraints that follow from the second law of thermodynamics. ${ }^{53,80-85}$ The most important of these is that all eigenvalues of the matrix $\mathbf{D}$ must be real and positive. As a consequence, $\operatorname{det}(\mathbf{D})>0$ and $\operatorname{tr}(\mathbf{D})>0$. One mathematical proof of this statement is based on the fact that the matrix $\mathbf{D}$ can be related to the symmetric matrix $\mathbf{L}$ consisting of the wellknown Onsager coefficients, ${ }^{56}$ for which $L_{i j}=L_{j i}$. The phenomenological coefficients $L_{k j}$ link the generalized forces $F_{j}$, for example, the gradient of chemical potential $\mu_{i}$, to the generalized fluxes $J_{k}$, for example, the diffusion flux. ${ }^{86,87} \mathrm{D}$ can be decomposed into the product of two positive definite matrixes, one of which is $\mathbf{L}^{53}$ Since all the eigenvalues of a positive definite matrix, by definition, are real and positive, the matrix $\mathbf{D}$, which is the product of two positive definite matrices, must also have only real, positive eigenvalues. ${ }^{81}$ Physically, a complex eigenvalue of $\mathbf{D}$ would result in oscillatory relaxation of any small perturbation to the equilibrium state, even in the absence of reaction.

In some works, in which the effect of cross-diffusion on pattern formation or wave propagation was investigated with the aid of eqn (3), this fundamental restriction on the diffusion matrix $\mathbf{D}$ has been ignored. ${ }^{35,36,88,89}$ As a result, surprising and probably physically unrealistic dynamic behaviors of the model RD systems were obtained. However, in biological, ecological and even social systems that can be described by eqn (3), the matrix D need not follow this thermodynamic restriction, since the diffusion processes, chemical or not, in these systems are driven by a source of external energy. The fundamental theoretical question that we pose, and address further below, is whether, in chemical systems far from equilibrium, the thermodynamic restriction on mutual diffusion coefficients, which derives from the linear phenomenological laws that hold close to equilibrium, can be violated due to interaction between chemical reactions and diffusion processes. This issue does not appear to have been considered in the theoretical literature, and measurements of diffusion coefficients in chemical systems far from equilibrium are lacking.

There are many methods for measuring diffusion coefficients in physicochemical systems. ${ }^{70}$ Three approaches, the optical interferometry technique, ${ }^{59}$ the diaphragm-cell technique, ${ }^{61}$ and the Taylor dispersion technique ${ }^{64-66}$ have been adapted for measuring cross-diffusion coefficients. Such powerful methods as dynamic light scattering (DLS) and pulsed NMR have not thus far been utilized to determine cross-diffusion coefficients. Pulsed NMR is used to measure self-diffusion coefficients, while DLS probably measures the smallest eigenvalue of the matrix $\mathbf{D} .{ }^{90}$ In ternary systems [particular in glycerol(0)-acetone(1)-water(2)] it is found that the diffusion coefficient $D_{\mathrm{m}}$ obtained from DLS experiments is not related to any of the diffusion coefficients $D_{i j}$ in the Fick's diffusion coefficient matrix $\mathbf{D},{ }^{90}$ but it does coincide with the lowest eigenvalue of $\mathbf{D}$. This result may be general, at least for molecules of similar size.

One of the most widely employed techniques for measuring diffusion coefficients is the Taylor dispersion method. Although it dates back over half a century, it is only in the past two decades 
that this technique has been adapted for measuring crossdiffusion in three- and even four-component systems. ${ }^{65,91-93}$ The Taylor method is based on the diffusive spreading of a drop of solution injected into a laminarly flowing stream of a mixture of the same components but with slightly different concentrations (see Fig. 1). A small volume of the perturbing solution is injected into the flowing eluent at the entrance of a long capillary tube. As it moves along the tube, the injected sample is deformed by the flow, the rate of which has a parabolic shape across the capillary, and by radial diffusion. The drop spreads out into a shape that can be fitted by a combination of $n$ Gaussian functions for an $(n+1)$-component system. The eluted peak, sometimes called the Taylor peak, is monitored by a suitable detector such as a flow-through spectrophotometer, refractive index detector (RID) or Raman spectrometer. ${ }^{93,94}$ The diffusion coefficients are calculated from the parameters of the Gaussian functions that fit the eluted peak. A typical peak is shown in Fig. 2.

In general, the cross-diffusion coefficients or off-diagonal elements of matrix $\mathbf{D}$ can be either positive or negative and can be quite large in certain cases. Three important mechanisms responsible for cross-diffusion are electrostatic interactions, excluded volume effects, and "complexation".

Cross diffusion for charged particles at very low concentrations (infinitely dilute solutions) can be described with the NernstHartley equations, ${ }^{70,95-97}$ valid when the off-diagonal Onsager coefficients are zero. These equations are often used, even at large concentrations of charged species, to provide at least a qualitative description of diffusion processes. ${ }^{27,29,98,99}$ The Nernst-Hartley equations are based on the hypothesis of electroneutrality: charged particles cannot move independently, since each volume element in the solution must be neutral. For a binary mixture with a univalent single electrolyte, for example, the Nernst-Hartley equation takes the form

$$
D_{ \pm}^{\infty}=2 D_{+}^{\infty} D_{-}^{\infty} /\left(D_{+}^{\infty}+D_{-}^{\infty}\right)
$$

where the superscript $\infty$ denotes the limit of infinite dilution, and $D_{ \pm}^{\infty}$ is the diffusion coefficient of an entire electrolyte molecule.

For ternary mixtures in which one component is a large molecule (e.g., a protein) and the other is salt, the NernstHartley equations are ${ }^{96}$

$$
D_{11}=D_{\mathrm{p}}\left[1+z_{\mathrm{p}}^{2} C_{1}\left(D_{\mathrm{c}}-D_{\mathrm{p}}\right) / Q\right]
$$

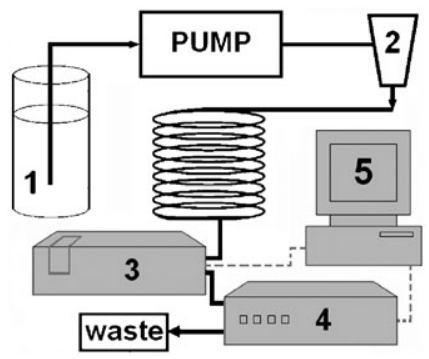

Fig. 1 Schematic drawing of an experimental setup for Taylor dispersion measurements: 1, reservoir containing the eluent; "pump", isocratic pump; 2, injector; the long $(30 \mathrm{~m})$ coiled Teflon tubing is connected to the outlet of the injector; 3, spectrophotometer; 4, refractive index detector (RID); 5, personal computer.

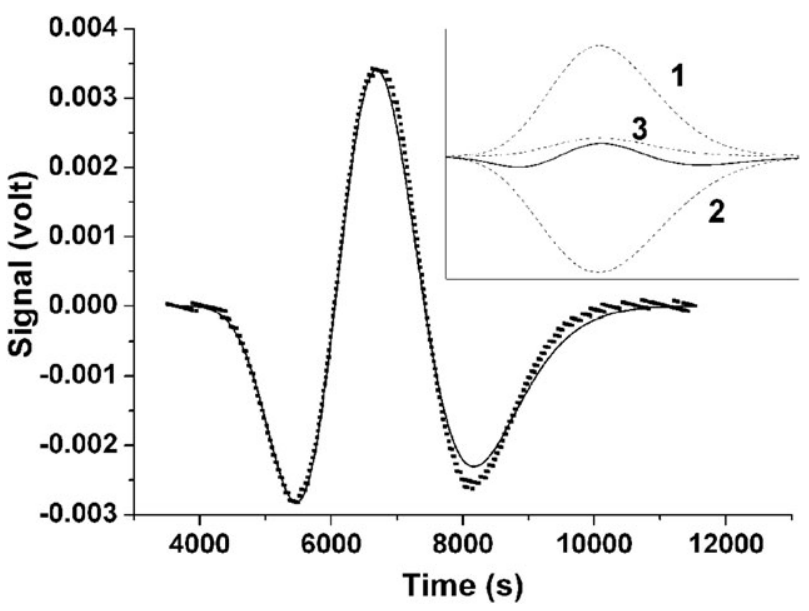

Fig. 2 Taylor dispersion peak for 4-component system water/AOT/ MA/octane. Injected samples contain an excess of MA (malonic acid). Dotted line is the experimental Taylor peak (signal) and solid line is the fitted curve. Inset shows the contribution of each component to the fitted (analytical) curve (solid line): curves 1, 2, and 3 correspond to the signals of $\mathrm{H}_{2} \mathrm{O}$, AOT (Aerosol OT, surfactant), and MA, respectively. From ref. 93.

$$
\begin{aligned}
& D_{12}=D_{\mathrm{p}} z_{\mathrm{p}} z_{\mathrm{M}} C_{1}\left(D_{\mathrm{c}}-D_{\mathrm{M}}\right) / Q \\
& D_{21}=D_{\mathrm{M}} z_{\mathrm{p}} z_{\mathrm{M}} C_{2}\left(D_{\mathrm{c}}-D_{\mathrm{p}}\right) / Q \\
& \left.D_{22}=D_{\mathrm{M}}\left[1+z_{\mathrm{M}} C_{2} C_{\mathrm{c}}-D_{\mathrm{M}}\right) / Q\right]
\end{aligned}
$$

where $D_{\mathrm{p}}, D_{\mathrm{M}}$, and $D_{\mathrm{c}}$ are the (tracer) diffusion coefficients of the macromolecule (cation or anion), the co-ion (e.g., $\mathrm{Na}^{+}$, $z_{\mathrm{M}}=1 ; \mathrm{Cl}^{-}, z_{\mathrm{M}}=-1 ; \mathrm{Mg}^{2+}, z_{\mathrm{M}}=2$, where $z_{\mathrm{M}}$ is the charge of the ion), and the common ion (e.g. chloride), respectively; and $z_{\mathrm{p}}$ is the charge, which depends on $\mathrm{pH}$ and on the macromolecule ( $z_{\mathrm{p}}$ and $z_{\mathrm{M}}$ have the same sign); $C_{1}$ is the concentration of macromolecule, $C_{2}$ is the concentration of co-ion, and $Q=z_{\mathrm{p}} C_{1}\left(D_{\mathrm{p}} z_{\mathrm{p}}+D_{\mathrm{c}}\right)+z_{\mathrm{M}} C_{2}\left(D_{\mathrm{M}} z_{\mathrm{M}}+D_{\mathrm{c}}\right)$. Eqn (8)-(11) are valid only for $z_{\mathrm{p}} C_{1}<<z_{\mathrm{M}} C_{2}$.

The excluded volume effect can be explained in terms of the effective concentration of component $i, c_{i}{ }^{*}$, given by

$$
c_{i}^{*}=c_{i} /\left(1-\phi_{k}\right), i \neq k
$$

Here $\phi_{k}$ is the volume fraction of component $k, \phi_{k}=V_{k} c_{k}$, where $V_{k}$ is the partial molar volume of $k$. Using mostly intuitive relations and assuming that, in the absence of interactions, the flux of component $i$ generated by the gradient $\nabla c_{k}$ should be the same of the flux of $i$ produced by its own concentration gradient $\nabla c_{i}^{*}$ :

$$
D_{i k} \nabla c_{k}=D_{i i} \nabla c_{i}^{*}
$$

the following relation between $D_{i k}$ and $D_{i i}$ was obtained: ${ }^{43,45}$

$$
D_{i k}=D_{i i} V_{k} c_{i} /\left(1-V_{k} c_{k}\right)^{2}(i \neq k)
$$

from which we see that $D_{i k}$ should be positive (assuming that $D_{i i}$ is positive).

Cross-diffusion can arise via complexation through a variety of physical interactions between species, like the entropic interaction in micelles or the weak interaction between enzyme and substrate or host-guest complexes. Suppose that two species $\mathrm{X}$ and $\mathrm{P}$ interact rapidly and reversibly so as to 
maintain local reaction equilibrium due to formation of the transient complex XP:

$$
\mathrm{X}+\mathrm{P} \leftrightarrow \mathrm{XP}
$$

Then the mutual diffusion coefficients for species $1=\mathrm{X}+\mathrm{XP}$ (total $\mathrm{X})$ and species $2=\mathrm{P}+\mathrm{XP}($ total $\mathrm{P})$ are given by ${ }^{100-104}$

$$
\begin{aligned}
& D_{11}=D_{\mathrm{X}}+\phi_{2}\left(D_{\mathrm{XP}}-D_{\mathrm{X}}\right) \\
& D_{12}=\phi_{1}\left(D_{\mathrm{XP}}-D_{\mathrm{X}}\right) \\
& D_{21}=\phi_{2}\left(D_{\mathrm{XP}}-D_{\mathrm{P}}\right) \\
& D_{22}=D_{\mathrm{P}}+\phi_{1}\left(D_{\mathrm{XP}}-D_{\mathrm{P}}\right)
\end{aligned}
$$

where $D_{\mathrm{A}}$ is the diffusion coefficient of species $\mathrm{A}(=\mathrm{X}, \mathrm{P}$, or $\mathrm{XP)}$ without any interaction and

$$
\begin{aligned}
& \phi_{1}=c_{\mathrm{X}} c_{\mathrm{XP}} /\left(c_{\mathrm{XP}} c_{\mathrm{P}}+c_{\mathrm{XP}} c_{\mathrm{X}}+c_{\mathrm{P}} c_{\mathrm{X}}\right) \\
& \phi_{2}=c_{\mathrm{P}} c_{\mathrm{XP}} /\left(c_{\mathrm{XP}} c_{\mathrm{P}}+c_{\mathrm{XP}} c_{\mathrm{X}}+c_{\mathrm{P}} c_{\mathrm{X}}\right)
\end{aligned}
$$

If for example $\mathrm{P}$ is a very large molecule (e.g., a polymer or starch bound to a gel), we can assume that $D_{\mathrm{P}}=D_{\mathrm{XP}}=0$ and eqn (16)-(19) give

$$
\begin{aligned}
& D_{11}=\left(1-\phi_{2}\right) D_{\mathrm{X}} \\
& D_{12}=-\phi_{1} D_{\mathrm{X}} \\
& D_{21}=D_{22}=0
\end{aligned}
$$

The cross-diffusion coefficients $D_{12}$ and $D_{21}$ are always negative or zero, since $D_{\mathrm{XP}}<D_{\mathrm{X}}$ and $D_{\mathrm{XP}}<D_{\mathrm{P}}$, for example, $D_{12}$ takes values between 0 and $-D_{\mathrm{X}}$. The negative sign of $D_{12}$ indicates that $\mathrm{X}$ is attracted toward higher concentrations of $\mathrm{P}$ as a result of the complexation reaction (15).

In an actual experimental system, any of these mechanisms is likely to interact with one or more other processes. In the following we summarize several results that illustrate different cases of cross-diffusion in physical-chemical systems without reaction. These experiments were performed either with the highly accurate Gouy or Rayleigh optical interferometry technique using a unique Gosting diffusiometer ${ }^{105}$ or by the Taylor method. We will see that cross-diffusion coefficients can be quite significant, even in apparently simple systems.

Salt-salt. Very careful investigation of cross-diffusion coefficients has been carried out in aqueous solutions of $\mathrm{NaCl}$ $\left(c_{1}\right)$ and $\mathrm{MgCl}_{2}\left(c_{2}\right)$, as well as in solutions of $\mathrm{NaCl}\left(c_{1}\right)$ and $\mathrm{Na}_{2} \mathrm{SO}_{4}\left(c_{2}\right) \cdot{ }^{40,106-109}$ For the $\mathrm{NaCl}-\mathrm{MgCl}_{2}$ system, at molar ratios $c_{1} / c_{2}>1$ (e.g., $\left.c_{1} / c_{2}=3\right),{ }^{40,107}$ the positive cross-term $D_{12}$ becomes quite large at moderate and high concentrations, and it can even exceed the diagonal coefficients $D_{11}$ and $D_{22}$.

$$
\begin{gathered}
\mathbf{D}=\left(\begin{array}{ll}
1.156 & 1.284 \\
0.131 & 0.751
\end{array}\right) \times 10^{-5} \mathrm{~cm}^{2} \mathrm{~s}^{-1} \\
\text { at } c_{1}=2.845 \mathrm{M}, c_{2}=0.948 \mathrm{M}
\end{gathered}
$$

However, when $c_{1} / c_{2} \leq 1,{ }^{108,109}$ both of the cross-diffusion coefficients $D_{12}$ and $D_{21}$ remain small. For the $\mathrm{NaCl}-\mathrm{Na}_{2} \mathrm{SO}_{4}$ system at a constant total molarity of $0.5 \mathrm{M},{ }^{106} D_{21}$, the cross diffusion coefficient of $\mathrm{Na}_{2} \mathrm{SO}_{4}$ due to a concentration gradient of $\mathrm{NaCl}$, was found to be negative, while $D_{12}$ is positive, in agreement with the ternary Nernst-Hartley eqn (8)-(11) for electrolytes.

Polymer-salt. A very large positive cross-diffusion coefficient $D_{21}$ was obtained for the aqueous ternary system lysozyme $\left(c_{1}\right)-\mathrm{MgCl}_{2}\left(c_{2}\right)$ at $c_{1}=0.5987 \mathrm{mM}$, and $c_{2}=0.9304 \mathrm{M}$ $\left(\mathrm{pH}=4.5,25^{\circ} \mathrm{C}\right)$ with an almost linear dependence of $D_{21}$ on $c_{2}{ }^{29}$ In units of $10^{-5} \mathrm{~cm}^{2} \mathrm{~s}^{-1}$, the authors found

$$
\mathbf{D}=\left(\begin{array}{cc}
0.0833 & 0.000145 \\
12.3 & 1.080
\end{array}\right)
$$

In that work, the diffusion of charged macromolecules in the presence of an electrolyte solution was discussed in terms of two models: (a) the Nernst-Hartley equations, ${ }^{96}$ applicable to an ideal dilute ternary solution with two ionic components, and; (b) the Stokes-Einstein equation, ${ }^{110}$ which describes the tracer diffusion coefficient (or mobility) of macromolecules in the limit of $c_{1} \rightarrow 0$. For both models, the diffusion coefficients are independent of the reference frame, because they apply at infinite dilution. The Nernst-Hartley equations are able to qualitatively describe the observed concentration dependences of the coefficients $D_{i j}$. A partial explanation of these $D_{i j}$ can be obtained with an excluded volume model, which we discuss in the next paragraph. Analogous results were obtained for a lysozyme chloride- $\mathrm{NaCl}$ system. ${ }^{87}$

Investigation of transport of electrolytes in poly(ethylene glycol) (PEG) revealed strong cross-diffusion due to excluded volume effects. ${ }^{42}$ For a PEG $2000\left(c_{1}\right)-\mathrm{NaCl}\left(c_{2}\right)-\mathrm{H}_{2} \mathrm{O}$ ternary system, at $c_{1}=0.1250 \mathrm{M}$ and $c_{2}=1.2485 \mathrm{M}$, it was found that (in units $10^{-5} \mathrm{~cm}^{2} \mathrm{~s}^{-1}$ )

$$
\mathbf{D}=\left(\begin{array}{cc}
0.23 & 0.0132 \\
3.49 & 0.654
\end{array}\right)
$$

with an almost linear dependence of $D_{21}$ on $c_{2}$. This behaviour is consistent with that observed in other PEG-NaCl- $\mathrm{H}_{2} \mathrm{O}$ systems. $^{47,48}$ The excluded-volume mechanism applied to the PEG-NaCl system assumes that the volume occupied by the large PEG molecules is unavailable to the salt ions, thus increasing the effective concentration of $\mathrm{NaCl}: c_{2}{ }^{*}=c_{2} /\left(1-\phi_{1}\right) \equiv c_{2} /\left(1-V_{1} c_{1}\right)$, where $V_{l}$ is the effective hydrodynamic volume of a PEG molecule. $^{45,47}$

Polymer-salt_1-salt_2. A more complex variant of the lysozyme- $\mathrm{NaCl}$ system consists of $\mathrm{H}_{3} \mathrm{PO}_{4}\left(c_{1}=0.1 \mathrm{M}\right)-\mathrm{KH}_{2} \mathrm{PO}_{4}$ $\left(c_{2}=0.1 \mathrm{M}\right)-\mathrm{BSA}\left(c_{3}=0.1 \mathrm{mM}\right)$-water( 0$)$, where BSA $=$ bovine

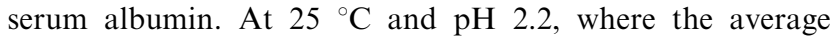
charge on the BSA species is +92 , the matrix of diffusion coefficients is found to be $\mathrm{e}^{111}$

$$
\mathrm{D}=\left(\begin{array}{ccc}
1.26 & -0.55 & -104 \\
-0.42 & 1.32 & 60 \\
-0.00013 & 0.00004 & 0.07
\end{array}\right) \times 10^{-5} \mathrm{~cm}^{2} \mathrm{~s}^{-1}
$$

A related system, ${ }^{111}$ which illustrates the strong dependence of the cross-diffusion coefficients on the polymer charge, consists of $\mathrm{KH}_{2}$ citrate $\left(c_{1}=0.1 \mathrm{M}\right)-\mathrm{K}_{2} \mathrm{Hcitrate}\left(c_{2}=0.1 \mathrm{M}\right)-\operatorname{BSA}\left(c_{3}=0.1 \mathrm{mM}\right)$. The isoelectric point for BSA is $\sim \mathrm{pH} 5.4 .{ }^{112}$ At $25{ }^{\circ} \mathrm{C}$ and $\mathrm{pH} 4.5$, where BSA has an average charge of +40

$$
\mathrm{D}=\left(\begin{array}{ccc}
0.66 & 0.08 & -12 \\
0.07 & 0.81 & 11 \\
-0.00002 & 0 & 0.061
\end{array}\right) \times 10^{-5} \mathrm{~cm}^{2} \mathrm{~s}^{-1}
$$


while at $\mathrm{pH} 5.9$, where the BSA charge is negative,

$$
\mathrm{D}=\left(\begin{array}{ccc}
0.67 & 0.06 & 36 \\
0.14 & 0.87 & -23 \\
0.00006 & 0.0001 & 0.064
\end{array}\right) \times 10^{-5} \mathrm{~cm}^{2} \mathrm{~s}^{-1}
$$

Note the change in sign of the elements $D_{i 3}$.

Leaist and $\mathrm{HaO}^{111}$ develop a simple procedure to estimate the multicomponent Fick diffusion coefficients $\left(D_{i k}\right)$ from the diffusion coefficients $D_{\mathrm{s}}$ of the species that appear in the NernstPlanck equation ${ }^{113}$

$$
j_{\mathrm{s}}=-D_{\mathrm{s}} \partial c_{\mathrm{s}} / \partial x+(F / R T) D_{\mathrm{s}} c_{\mathrm{s}} z_{\mathrm{s}} E
$$

where $j_{\mathrm{s}}$ is the molar flux of species $s$ in the $x$-direction, and $D_{\mathrm{s}}$, $c_{\mathrm{s}}$, and $z_{\mathrm{s}}$ are the diffusion coefficient, concentration and charge of that species; $F$ is the Faraday constant, $R$ the gas constant and $T$ the temperature. The diffusion of the ionic species is coupled to the electric field $E$, which slows down the more mobile ions and speeds up the slower ones, so that all parts of the solution remain electrically neutral. Linking the two sets of equations, Fick and Nernst-Planck, allows the better features of both treatments to be exploited.

Micelle-salt. A large negative cross diffusion coefficient was found in a micelle system with salt: DSDMAOH (distearyldimethylammonium hydroxide) $\left(c_{1}\right)-\mathrm{NaCl}\left(c_{2}\right)$ at $\mathrm{pH} 7.5$ with $c_{1}=40 \mathrm{mM}$, and $c_{2}=5 \mathrm{mM}$ or $15 \mathrm{mM}^{27}$

$$
\begin{aligned}
& \mathbf{D}=\left(\begin{array}{cc}
0.349 & 0.006 \\
-7.2 & 1.5
\end{array}\right)\left(c_{2}=5 \mathrm{mM}\right), \\
& \mathbf{D}=\left(\begin{array}{ll}
0.349 & 0.014 \\
-2.5 & 1.54
\end{array}\right)\left(c_{2}=15 \mathrm{mM}\right)
\end{aligned}
$$

in units of $10^{-5} \mathrm{~cm}^{2} \mathrm{~s}^{-1}$

Another experiment with a micelle-salt system, SDS (sodium dodecyl sulfate) $\left(c_{1}=0.1 \mathrm{M}\right)-\mathrm{NaCl}\left(c_{2}=0.1 \mathrm{M}\right)^{114}$ gives negative $D_{12}$.

Polymer-micelle. Large positive cross-diffusion coefficients were observed in a mixture of aqueous solutions of the polymer PEG3400 [poly(ethyleneglycol) with average molecular weight $\left.3400 \mathrm{~g} \mathrm{~mol}^{-1}\right]\left(c_{1}\right)$ with micelles of $\operatorname{SDS}\left(c_{2}\right) \cdot{ }^{43}$ For example, at $c_{1}=0.00296 \mathrm{M}$ and $c_{2}=0.2501 \mathrm{M}$, the following coefficients (in units of $10^{-5} \mathrm{~cm}^{2} \mathrm{~s}^{-1}$ ) were obtained

$$
\mathbf{D}=\left(\begin{array}{ll}
0.17 & 0.01 \\
5.87 & 0.74
\end{array}\right)
$$

The large coefficient $D_{21}$ is almost linearly proportional to $c_{2}$. Analogous results (large positive $D_{21}$ ) were obtained with aqueous solutions of poly(vinylpyrrolidone) (PVP, $24000 \mathrm{~g} \mathrm{~mol}^{-1}$ ) and sodium octyl sulfonate. ${ }^{115}$ The large cross-diffusion $\left(D_{21}>>D_{22}, D_{11}\right)$ was interpreted as a result of electrostatic coupling, which prevents ionic species from moving independently due to electroneutrality.

Reverse micelles. Very large positive and negative crossdiffusion coefficients have been found in three-component water-in-oil AOT microemulsions consisting of water(l)AOT(2)-heptane $28,116,117$ and water(1)-AOT(2)-octane, and in the analogous four-component systems water(1)-AOT(2)ferroin(3)-octane and water(1)-AOT(2)-malonic acid(3)-octane. ${ }^{93}$ The large positive $D_{12}$ grows almost linearly with the radius of the water nanodroplets, which is proportional to the ratio $\omega=c_{1} / c_{2}$, and can be approximately expressed as ${ }^{28,116}$

$$
D_{k l}=c_{k}\left(\partial D_{k}^{*} / \partial c_{l}\right),(l \neq k)
$$

where $D_{k}^{*}$ is the intradiffusion coefficient, approximately equal in this case to the diffusion coefficient $D_{\mathrm{d}}$ of an entire droplet, and consequently all $D_{k}^{*}$ are equal.

The quaternary diffusion coefficients (in $10^{-6} \mathrm{~cm}^{2} \mathrm{~s}^{-1}$ ) for the water(1)/AOT(2)/MA(3)/octane system at $\omega=11.84, \varphi_{\mathrm{d}}=0.18$, [MA] $=0.032 \mathrm{M}$, and $T=23{ }^{\circ} \mathrm{C}$ are ${ }^{93}$

$$
\mathbf{D}=\left(\begin{array}{ccc}
0.57 & 6 & 8 \\
-0.012 & 1.6 & 1.8 \\
-0.0006 & -0.08 & 0.56
\end{array}\right)
$$

In the water(1)-AOT(2)-ferroin(3)-octane system at $\omega=11.84$, $\varphi_{\mathrm{d}}=0.18$, [ferroin] $=1.61 \times 10^{-5} \mathrm{M}$, and $T=23{ }^{\circ} \mathrm{C}^{93}$

$$
\mathbf{D}=\left(\begin{array}{ccc}
0.5 & 5.2 & -45 \\
-0.012 & 1.2 & -12 \\
0.00022 & 0.0032 & 0.4
\end{array}\right) \times 10^{-6} \mathrm{~cm}^{2} \mathrm{~s}^{-1}
$$

The change in sign of the coefficients $D_{13}$ and $D_{23}$ when MA is replaced by ferroin can be explained by combining eqn (26) with data from dynamic light scattering experiments that reveal that the radius of water nanodroplets decreases slightly if we add MA and increases (because of cluster formation) if we add ferroin to pure reverse micelles. These changes in the radius and corresponding changes in the diffusion coefficients of the droplets $\left(D_{k}{ }^{*}\right)$ result in $\partial D_{k} * / \partial c_{l}$ being positive for MA and negative for ferroin, yielding the observed signs of $D_{i 3}$.

\section{Cross-diffusion on surfaces and in porous materials}

There is no direct method to measure cross-diffusion coefficients on surfaces, where many patterns in reaction-diffusion systems have been found. ${ }^{118-124}$ Experimental results on cross-diffusion in porous materials or in gels are scarce. For example, Sasaki and Maeda found that cross-diffusion between $\mathrm{NaNO}_{3}(1)$ and $\mathrm{NaCl}$ (2) in porous silica spheres was quite small: $D_{21}=0$ and $D_{12} / D_{22}=-0.022$. The negative $D_{12}$ was attributed to electrostatic effects (Nernst-Hartley equation). In general, all diffusion coefficients in a porous material are smaller than in the corresponding aqueous solution. There are several studies of selfdiffusion coefficients in gels using pulsed NMR. Some of them, for example the diffusion of SDS in a cross-linked gel of ethyl(hydroxyethyl) cellulose, reveal anomalous (non-Gaussian) diffusion, probably due to inhomogeneities in the gel. ${ }^{126}$ There is also considerable evidence that the pore size has a significant effect on diffusion coefficients. ${ }^{125,127-129}$

Theoretical considerations suggest that there should be large cross-diffusion coefficients as a result of excluded volume effects. For example, if we have two species A and B adsorbed on a surface and the coverage (fraction of the surface occupied) by A, $\phi_{\mathrm{A}}$, is significant, it is clear that the diffusion of $\mathrm{B}$ must decrease with $\phi_{\mathrm{A}}$. An analogous situation must occur in porous materials, for example in the case when the size of the larger species is close to the diameter of the pores. Blocking of the pores by the larger particles will hinder the diffusion of the smaller ones. This effect was recently demonstrated by Shaw et al. ${ }^{130}$ in experiments and simulations with a mixture of small 
and large spherical particles in a chamber divided by a membrane with asymmetric pores.

Recently, Stojanovic and coworkers have shown that complex molecules containing ribozymes can diffuse along a path covered with immobilized oligonucleotide substrates. ${ }^{131}$ These "walkers" do a remarkable job of "keeping to the path" as a result of cross-diffusion due to transient formation of a substrate-enzyme complex.

\section{Cross-diffusion in reaction-diffusion systems}

A number of theoretical papers discuss pattern formation in reaction-diffusion (RD) systems with cross-diffusion. ${ }^{132-142}$ Almost all these works include nonlinear kinetic terms describing autocatalysis and/or negative feedback in their models. A single study finds that with appropriate crossdiffusion coefficients, linear reaction terms are sufficient to produce pattern formation. ${ }^{143}$ Before analyzing the role of cross-diffusion in actual RD systems, we consider several examples of related systems that can be described by analogous models.

\section{A "Cross-diffusion" in living systems: chemotaxis and ecology}

While the notion that the spatial distribution of one species may affect the motion of other species may seem less than intuitive in chemical systems, such behaviour would appear to be the norm in living systems. Phenomena of this type have been more thoroughly studied in the biological than in the chemical literature, and we mention here a few examples.

Chemotaxis, the phenomenon in which cells direct their motion toward or away from higher concentrations of chemical species, sometimes referred to as chemoattractants or chemorepellants, is perhaps the clearest example of "crossdiffusion" in biology. Bacteria like E. coli alternately swim in straight lines and "tumble," changing their direction in preparation for their next straight-line excursion. The motion resembles a diffusive random walk, and in the absence of chemical gradients, a bacterium obeys the same laws as a diffusing particle. The mechanism of tumbling involves a change in the direction of rotation of the bacterial flagella. When gradients of attractants or repellants are present, the bacterium adjusts its frequency of tumbling, changing direction less often if it senses that it is swimming toward higher attractant or lower repellant concentrations. ${ }^{144}$ The result is a net motion, chemotaxis, up a gradient of attractant, for example. Viewed as a form of cross-diffusion, the phenomenon is characterized by a negative $D_{\text {bacterium-attractant }}$ and a positive $D_{\text {bacterium-repellant }}$. Chemotaxis of $E$. coli gives rise to striking patterns like the ones shown in Fig. 3. ${ }^{32}$ A number of models have been developed and analyzed that describe chemotaxis in terms of diffusion, including cross-diffusion, of cells and chemoattractants. $^{145,146}$

Ecology provides many examples of pattern formation arising from the motion of one species in response to gradients in the abundance of other species. Meron and coworkers ${ }^{147-150}$ have modeled the development of vegetation patterns in water-limited regions by taking into account cross-diffusive terms between the biomass density and the groundwater density, where water plays the role of a "chemoattractant" for plants.

As a final example, we observe that predator-prey systems, the inspiration for Lotka's early model ${ }^{151}$ of autocatalysis and chemical oscillation, are naturally described in terms of crossdiffusion. Clearly, predators will tend to gravitate toward higher concentrations of prey, while prey will preferentially move toward regions where predators are rare. Models of predator-prey systems with cross-diffusion have been extensively analyzed in the literature, though often with respect to their mathematical properties rather than to provide insight into the kinds of patterns that can emerge.

del-Castillo-Negrete et al. ${ }^{135,152}$ examined a two-variable model with cross-diffusion relevant to both plasma physics and predator-prey population dynamics,

$$
\begin{aligned}
& \partial \varepsilon / \partial t=\varepsilon-\varepsilon^{2}-\sigma^{2} \varepsilon+\nabla(d+D \varepsilon) \nabla \varepsilon \\
& \partial \sigma / \partial t=-\mu \sigma+\alpha \sigma \varepsilon+\nabla(d+D \varepsilon) \nabla \sigma+\nabla D \sigma \nabla \varepsilon
\end{aligned}
$$

They showed that positive cross-diffusion $(D>0)$ induces instability in one of four homogeneous steady states and can give rise to front propagation between two of the steady states. Two fronts can collide to produce a stationary kink-state. Segregated states, the analog of Turing patterns in chemical systems, were also obtained in this model due to cross-diffusion.

\section{B "Cross-diffusion" in social systems}

Certain interactions between human beings also appear to be determined by behaviours that resemble cross-diffusion. People may prefer to live, for example, near others of the same ethnic group or social class or to avoid members of certain groups. The phenomenon of "white flight," in which members of the white middle or working classes moved away from
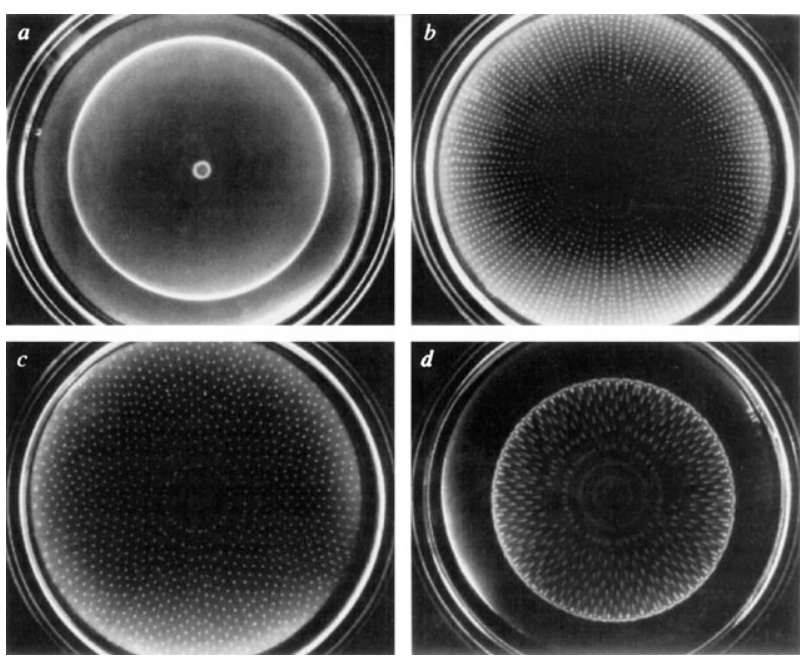

Fig. 3 Patterns generated by chemotactic E. coli bacteria in the presence of succinate, a chemoattractant. (a) swarm ring in $1 \mathrm{mM}$ succinate; (b) spots on a pseudo-rectangular lattice in $2 \mathrm{mM}$ succinate; (c) spots on a pseudo-hexagonal lattice in $3 \mathrm{mM}$ succinate; (d) spots with tails on a pseudo-hexagonal lattice in $3 \mathrm{mM}$ succinate with a mutant strain. From ref. 32. 
neighborhoods that became racially desegregated in the United Sates after World War II, causing resegregation, is one example of such behaviour.

Epstein ${ }^{153}$ has examined the illicit drug trade by introducing cross-diffusion terms into a standard model from epidemiology. Defining S as "susceptibles" (ordinary citizens), I as "infectives" (drug users/dealers) and L as law enforcement personnel, he describes the evolution of these three subgroups on a one-dimensional "street" by the set of equations

$$
\begin{aligned}
\partial \mathrm{S} / \partial t= & -\beta \mathrm{SI}+\mu \mathrm{S}+D_{\mathrm{SS}} \partial^{2} \mathrm{~S} / \partial x^{2} \\
\partial \mathrm{I} / \partial t= & \beta \mathrm{SI}-\gamma \mathrm{IL}+D_{\mathrm{IS}} \partial^{2} \mathrm{~S} / \partial x^{2}+D_{\mathrm{IL}} \partial^{2} \mathrm{~L} / \partial x^{2} \\
& +D_{\mathrm{II}} \partial^{2} \mathrm{I} / \partial x^{2} \\
\partial \mathrm{L} / \partial t= & \xi \mathrm{SIL}-b \mathrm{~L}+D_{\mathrm{LI}} \partial^{2} \mathrm{I} / \partial x^{2}+D_{\mathrm{LL}} \partial^{2} \mathrm{~L} / \partial x^{2}
\end{aligned}
$$

$D_{\mathrm{IS}}$ and $D_{\mathrm{LI}}$ are taken to be negative to capture the fact that infectives gravitate toward susceptibles and police move toward higher concentrations of law-breakers, while $D_{\mathrm{IL}}$ is positive, meaning that criminals seek to avoid law enforcement personnel. The model, dubbed "crimo-taxis" by its creator, predicts various kinds of pattern formation, with potential implications for policy makers.

\section{C Cross-diffusion in physicochemical systems}

There at least three classes of patterns found in physicochemical RD systems that may arise from cross-diffusion. The first group involves phase-separating mixtures of two polymers A and B with which undergo a photoinduced reversible reaction between them. ${ }^{154-162}$ Since phase separation shares key characteristics with cross-diffusion, we will examine these experiments below when we analyze patterns in simple systems.

The second category consists of patterns found in catalytic surface reactions. ${ }^{16-169}$ In general, the competition of species for adsorbed surface sites gives rise to cross-diffusion effects, which have been largely neglected in the literature to date. More specifically, adsorption of non-reactive potassium atoms on a catalytic $\mathrm{Rh}(110)$ surface promotes Turing-like patterns in the reaction between adsorbed $\mathrm{O}_{2}$ and $\mathrm{H}_{2}{ }^{163}$ Though the authors do not invoke cross-diffusion to explain their patterns, it seems likely that cross-diffusion due to excluded volume effects and/or interactions between $\mathrm{K}$ and $\mathrm{O}$ atoms plays a major role in generating these patterns. This effect of nonreactive potassium atoms provides an experimental example of the idea we introduce later that nonreactive species can affect the diffusion fluxes of reactive species through cross-diffusion, thereby inducing Turing or wave instabilities.

The third group of patterns occurs in the BZ-AOT system. ${ }^{170-172}$ We recently found that cross-diffusion in BZ-AOT microemulsions is quite significant and may therefore be responsible for many of the wealth of patterns observed in this system. $^{93}$ A sampling of BZ-AOT patterns is shown in Fig. 4, where we have grouped the most important classes of patterns: wave patterns (upper row and right column), Turing patterns (three left snapshots in the second row), patterns originating from a wave instability (third row), and localized patterns (three left snapshots in the last row). We will use models of the BZ-AOT system when we analyze the possible role of cross-diffusion in pattern formation in the following sections.

\section{Theoretical analysis of cross-diffusion with reactions}

We consider a general RD model with the following structure

$$
\begin{aligned}
\left(\begin{array}{c}
\partial c_{1} / \partial t \\
\ldots \\
\partial c_{n} / \partial t \\
\partial s_{n+1} / \partial t \\
\partial s_{n+2} / \partial t
\end{array}\right)=\left(\begin{array}{c}
R_{1}\left(c_{1}, c_{2}, \ldots, c_{n}\right) \\
\ldots \\
R_{n}\left(c_{1}, c_{2}, \ldots, c_{n}\right) \\
0 \\
\\
0
\end{array}\right) \\
+(\nabla)\left(\begin{array}{ccccc}
D_{11} & \ldots & D_{1 n} & D_{1(n+1)} & D_{1(n+2)} \\
\ldots & \ldots & \ldots & \ldots & \ldots \\
D_{n 1} & \ldots & D_{n n} & D_{n(n+1)} & D_{n(n+2)} \\
D_{(n+1) 1} & \ldots & D_{(n+1) n} & D_{(n+1)(n+1)} & D_{(n+1)(n+2)} \\
D_{(n+2) 1} & \ldots & D_{(n+2) n} & D_{(n+2)(n+1)} & D_{(n+2)(n+2)}
\end{array}\right)\left(\begin{array}{c}
\nabla c_{1} \\
\ldots \\
\nabla c_{n} \\
\nabla s_{1} \\
\nabla s_{2}
\end{array}\right)
\end{aligned}
$$

which is an extended form of eqn (3). The concentrations $c_{i}$ and $s_{n+j}$ denote reactive and nonreactive species, respectively. All elements of the matrix $\mathbf{D}$ may depend on the $c_{i}$ and the $s_{n+j}$ and on the reaction rate constants included in the reaction terms $R_{\mathrm{i}}$. The dependence of the off-diagonal elements $D_{i j}$ on concentrations was discussed in section 2. In particular, if $c_{i}$ tends to 0 , then $D_{i j} \rightarrow 0$, since there cannot be a flux of species $i$ if $c_{i}=0$ regardless of the gradient of species $j$. Often, $D_{i j}$ depends linearly, or nearly linearly, on $c_{i}$.

The notion that $D_{i j}$ may depend on the rate constants is not intuitively obvious, but it does follow from thermodynamic considerations about the chemical potential and the chemical affinity $\mathscr{A} .^{86,173}$ As was shown by Lefever et al., ${ }^{173}$ the Duhem-Jouget theorem implies that $\mathscr{A}$ determines both the diffusion flux and the chemical reaction rate, so that these quantities cannot be independent of each other. We show in the next section that if we assume that $D_{i j}$ and the rate constants $k_{i}$ in the reversible reaction (33)

$$
\mathrm{A} \leftrightarrow \mathrm{B}
$$

can be set independently, then an apparent violation of the second law of thermodynamics can occur. It was also shown theoretically, using a stochastic model of a reactive Lorentz gas, that reaction (33) with a catalyst can induce cross-diffusion between species A and B. ${ }^{174}$ This result characterizes exactly how a chemical reaction can affect cross-diffusion. Note that eqn (1) demonstrates the opposite effect, i.e., how diffusion affects rate constants.

\section{A Simple linear systems}

Consider eqn (32) in its simplest form obtained from reaction (33) with rate constants $k_{1}$ and $k_{2}$ for the forward and reverse reactions, respectively. The matrix $\mathbf{D}$ of 

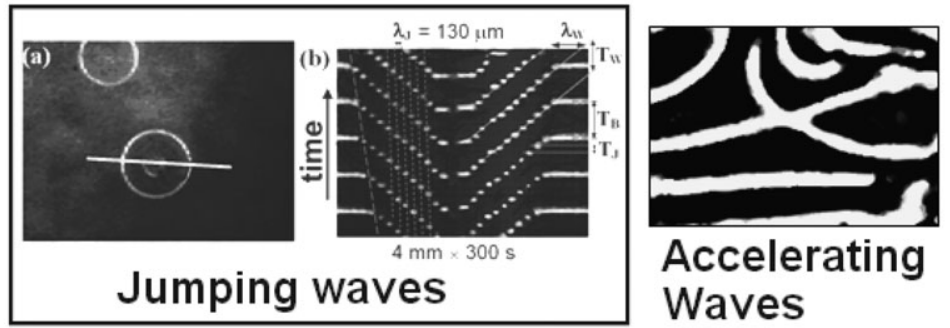

Accelerating Waves

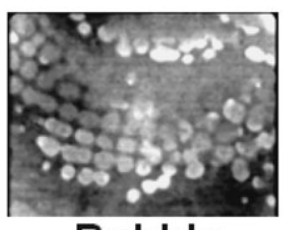

\section{Bubble}

waves
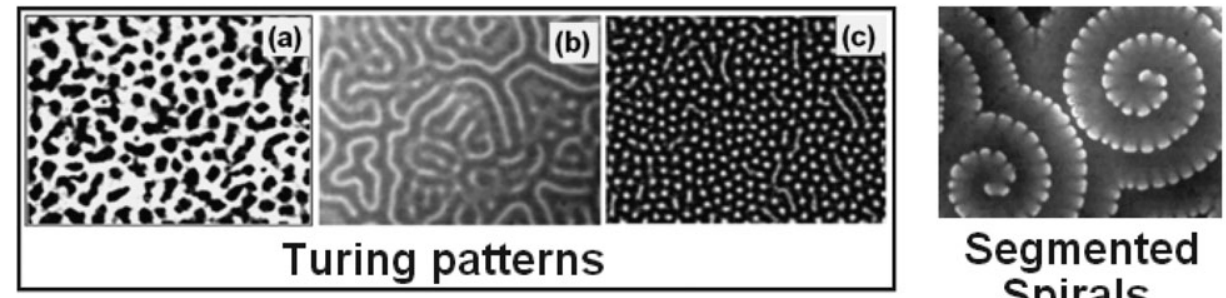

Segmented Spirals
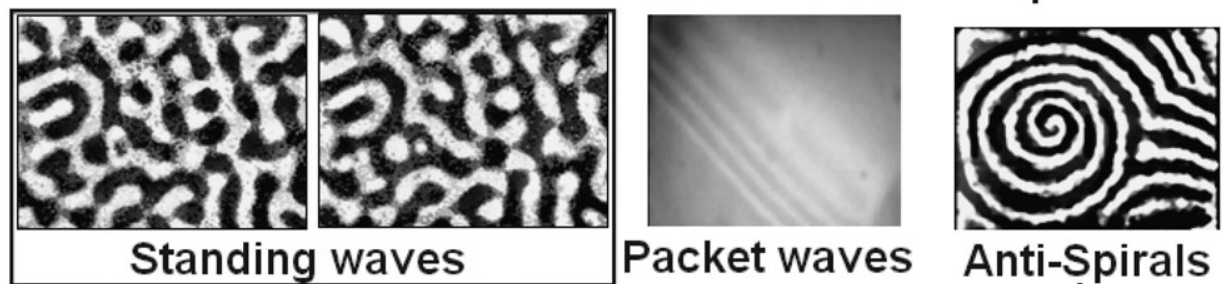

Packet waves

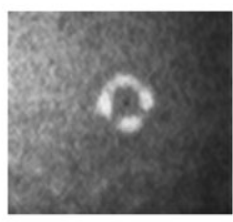

Oscillon

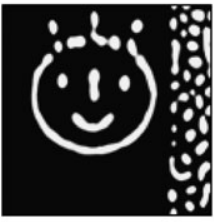

Chemical memory

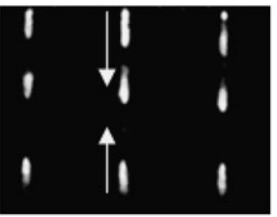

Localized waves
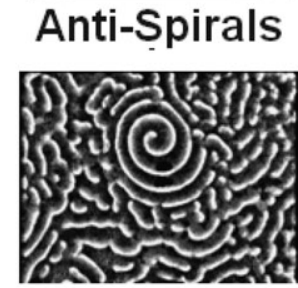

Spiral \& Chaos

Fig. 4 Several types of patterns found in the BZ-AOT system. Jumping waves are shown as a snapshot (a) and a space-time plot (b) across the white line shown in (a). Turing patterns are represented by three different types of structures: "black spots", "labyrinth", and "white spots". Standing waves are exhibited as two anti-phase snapshots. Localized structures are "oscillon", "chemical memory", and "localized waves" (arrows show direction of wave propagation).

diffusion coefficients has elements $D_{i j}$, where 1 corresponds to A and 2 to B. No nonreactive species are present. First, suppose that $k_{1}=k_{2}=0$. If we apply a small $\delta$-function perturbation of $\mathrm{A}$ at $x=x_{0}$ in a system with one spatial dimension (1D), then the root mean square displacement of $\mathrm{A}$, $\left\langle x_{\mathrm{A}}{ }^{2}\right\rangle^{1 / 2}$ with $D_{12}=D_{21}=0$ is determined by

$$
\begin{aligned}
\left\langle x_{A}^{2}\right\rangle & =\int_{-\infty}^{\infty}\left(x-x_{0}\right)^{2} \frac{1}{2 \sqrt{\pi D_{11} t}} \exp \left(-\frac{\left(x-x_{0}\right)^{2}}{4 D_{11} t}\right) \mathrm{d} x \\
& =2 D_{11} t
\end{aligned}
$$

There is no displacement of $\mathrm{B},\left\langle x_{\mathrm{B}}{ }^{2}\right\rangle=0$.

If we allow $D_{12}$ and/or $D_{21}$ to be non-zero, then the solution of the coupled diffusion equations

$$
\begin{aligned}
& \frac{\partial c_{1}}{\partial t}=D_{11} \frac{\partial^{2} c_{1}}{\partial x^{2}}+D_{12} \frac{\partial^{2} c_{2}}{\partial x^{2}} \\
& \frac{\partial c_{2}}{\partial t}=D_{21} \frac{\partial^{2} c_{1}}{\partial x^{2}}+D_{22} \frac{\partial^{2} c_{2}}{\partial x^{2}}
\end{aligned}
$$

is 93

$$
\begin{aligned}
& c_{1}=A_{11} G_{1}+A_{12} G_{2} \\
& c_{2}=A_{21} G_{1}+A_{22} G_{2}
\end{aligned}
$$

where

$$
G_{i}=\frac{1}{2 \sqrt{\pi \sigma_{i} t}} \exp \left(-\frac{\left(x_{0}-x\right)^{2}}{4 \sigma_{i} t}\right)
$$

$\sigma_{1}$ and $\sigma_{2}$ are the eigenvalues of the matrix $\mathbf{D}$ and

$$
\begin{aligned}
& A_{11}=\left[P_{1}\left(D_{11}-\sigma_{2}\right)+P_{2} D_{12}\right] /\left(\sigma_{1}-\sigma_{2}\right) \\
& A_{12}=\left[P_{1}\left(\sigma_{1}-D_{11}\right)-P_{2} D_{12}\right] /\left(\sigma_{1}-\sigma_{2}\right) \\
& A_{21}=\left[P_{2}\left(\sigma_{2}-D_{22}\right)-P_{1} D_{21}\right] /\left(\sigma_{2}-\sigma_{1}\right) \\
& A_{22}=\left[P_{2}\left(D_{22}-\sigma_{1}\right)+P_{1} D_{21}\right] /\left(\sigma_{2}-\sigma_{1}\right)
\end{aligned}
$$

with initial conditions (at $t=0$ and $x=x_{0}$ ) $A_{11}+A_{12}=P_{1}$ and $A_{21}+A_{22}=P_{2}$. Consider the case of $P_{2}=0$, i.e., 
a $\delta$-function perturbation of $\mathrm{A}$. The mean square displacements of $\mathrm{A}\left(=c_{1}\right)$ and $\mathrm{B}\left(=c_{2}\right)$ are now given by

$$
\begin{aligned}
& \left\langle x_{A}^{2}\right\rangle=\int_{-\infty}^{\infty}\left(x-x_{0}\right)^{2}\left(A_{11} G_{1}+A_{12} G_{2}\right) \mathrm{d} x=2 P_{1} D_{11} t \\
& \left\langle x_{B}^{2}\right\rangle=\int_{-\infty}^{\infty}\left(x-x_{0}\right)^{2}\left(A_{21} G_{1}+A_{22} G_{2}\right) \mathrm{d} x=2 P_{1} D_{21} t
\end{aligned}
$$

We see that species B has a displacement proportional to $D_{21}{ }^{1 / 2}$, while the displacement of $\mathrm{A}$ is the same as in the previous case without cross-diffusion (for $P_{1}=1$ ). Thus, if $P_{2}=0$, then the presence of species $\mathrm{B}$ does not affect the diffusion of species $\mathrm{A}$ even at non-zero $D_{12}$.

Consider another limiting case with $D_{12}=0, D_{21} \neq 0$, and arbitrary initial perturbations $P_{1}$ and $P_{2}$. Then $\sigma_{1}=D_{11}$, $\sigma_{2}=D_{22}$, and eqn (40)-(43) give $A_{11}=P_{1}, A_{12}=0$, $A_{21}=-P_{1} D_{21} /\left(D_{22}-D_{11}\right)$, and $A_{22}=P_{2}-A_{21}$. The mean square displacements of $\mathrm{A}$ and $\mathrm{B}$ are then given by

$$
\begin{aligned}
& \left\langle x_{A}{ }^{2}\right\rangle=2 P_{1} D_{11} t \\
& \left\langle x_{\mathrm{B}}{ }^{2}\right\rangle=2\left(P_{1} D_{21}+P_{2} D_{22}\right) t
\end{aligned}
$$

We see that species $B$ again does not affect species A, now because $D_{12}=0$. The displacement of B now depends on the sum $\left(P_{1} D_{21}+P_{2} D_{22}\right)$. These simple examples are meant to give some intuitive feeling about the properties of crossdiffusion.

We next introduce rate constants $k_{1}$ and $k_{2}$ and consider a $\delta$-function perturbation (at $x=x_{0}$ ) of the equilibrium steady state $\left(a_{\mathrm{ss}} k_{1}=b_{\mathrm{ss}} k_{2}\right)$; for simplicity, we apply an "equilibrium" perturbation, i.e., $P_{1} k_{1}=P_{2} k_{2}$. We can calculate the mean square displacements $\left\langle x_{\mathrm{A}}{ }^{2}\right\rangle$ and $\left\langle x_{\mathrm{B}}{ }^{2}\right\rangle$ as a function of $t$ to extract the effective diffusion coefficients, $D_{\mathrm{A}}=\left\langle x_{\mathrm{A}}{ }^{2}\right\rangle /\left(2 P_{1} t\right)$ and $D_{\mathrm{B}}=\left\langle x_{\mathrm{B}}{ }^{2}\right\rangle /\left(2 P_{2} t\right)$. For small $t$, i.e., when $t<<1 / \max \left(k_{1}, k_{2}\right)$, the diffusion process proceeds essentially as in the case $k_{1}=k_{2}=0$. For large $t$, however, when $t>>1 / \min \left(k_{1}, k_{2}\right)$, the situation is quite different. The effective diffusion coefficients are equal to each other and given by:

$$
\begin{aligned}
D^{*}=D_{\mathrm{A}}=D_{\mathrm{B}}= & \left(D_{21} k_{2}+D_{12} k_{1}+D_{11} k_{2}+D_{22} k_{1}\right) / \\
& \left(k_{1}+k_{2}\right)
\end{aligned}
$$

Note that this result is independent of the type of $\delta$-function perturbation ("equilibrium" or not), since at large $t$ equilibrium is established. If $D_{12}$ or $D_{21}$ is negative and $D_{12} / D_{22}<-1$ or $D_{21} / D_{11}<-1, D^{*}$ crosses zero and becomes negative with increasing $k_{1}$ or $k_{2}$, respectively. The condition

$$
D_{k} \equiv D_{21} k_{2}+D_{12} k_{1}+D_{11} k_{2}+D_{22} k_{1}=0
$$

marks the onset of Turing instability. Physically, condition (49) can be interpreted as follows. Suppose we start with small perturbation in $\mathrm{B}$ and $D_{21}=0$ (for simplicity), then $\mathrm{B}$ tends to diffuse away at a rate that depends on $D_{22}$, but because $D_{12}<0$, the perturbation in $\mathrm{B}$ also induces a flux of A directed toward the perturbation. If $k_{1}$ is large enough and $-D_{12}>D_{22}$ [condition (49) is fulfilled], this incoming A will be converted to B so rapidly that it will negate the outgoing flux of $\mathrm{B}$, and there will be no net diffusion of B away from the perturbation. However $D_{k}$ cannot be negative, since Turing patterns would then emerge in the closed equilibrium system (33), which is forbidden by thermodynamics. Condition (49) thus implies that there must be some co-dependence between the diffusion and reaction rate constants for reaction (33). For example, at large $k_{1}\left(k_{2}\right), D_{22}$ $\left(D_{11}\right)$ must be larger than $-D_{12}\left(-D_{21}\right)$.

Condition (49) can also be obtained from a linear stability analysis of the system (50)-(51).

$$
\begin{aligned}
& \partial a / \partial t=-k_{1} a+k_{2} b+D_{11} \nabla^{2} a+\operatorname{div}\left(D_{12} \nabla b\right) \\
& \partial b / \partial t=k_{1} a-k_{2} b+\operatorname{div}\left(D_{21} \nabla a\right)+D_{22} \nabla^{2} b
\end{aligned}
$$

where $a=[\mathrm{A}]$ and $b=[\mathrm{B}]$. To carry out the linear stability analysis, we must, in general, take into account the dependence of the cross-diffusion coefficients on $a$ and $b$. For example,

$$
\operatorname{div}\left(D_{12} \nabla b\right)=D_{12} \nabla^{2} b+\left(\mathrm{d} D_{12} / \mathrm{d} a\right) \nabla a \nabla b
$$

However, the last term in eqn (52) does not contribute to the linearized equations. Therefore the characteristic matrix $\mathbf{M}=\mathbf{J}-k^{2} \mathbf{D}$ (where $\mathbf{J}$ is the Jacobian for the linearized system, $\mathbf{D}$ is the diffusion matrix, and $k$ is the wavenumber) has the form

$$
\mathbf{M}=\left(\begin{array}{cc}
-k_{1}-k^{2} D_{11} & k_{2}-k^{2} D_{12} \\
k_{1}-k^{2} D_{21} & -k_{2}-k^{2} D_{22}
\end{array}\right)
$$

Turing instability for system (50), (51) occurs if ${ }^{175}$

$$
\operatorname{det}(\mathbf{M})<0
$$

The inequality (54) can be rewritten as:

$$
\begin{aligned}
\operatorname{det}(\mathbf{M})= & \operatorname{det}(\mathbf{D})\left(k^{2}\right)^{2}+\left(D_{21} k_{2}+D_{12} k_{1}\right. \\
& \left.+D_{11} k_{2}+D_{22} k_{1}\right) k^{2}+\operatorname{det}(\mathbf{J})<0
\end{aligned}
$$

Since $\operatorname{det}(\mathbf{J})=0$ in this case, and $\operatorname{det}(\mathbf{D})$ is always positive, then Turing instability requires $D_{k}<0$ (cf. eqn (49)).

Note that in open linear systems like

$$
\mathrm{A}_{0} \rightarrow \mathrm{A} \leftrightarrow \mathrm{B} \rightarrow 0
$$

considered by Almirantis and Papageorgiou, ${ }^{143}$ Turing patterns are also impossible, since the parametric region for the required cross-diffusion coefficients is even narrower than that for the corresponding closed system (33) with the same constants $k_{1}$, $k_{2}, D_{11}$, and $D_{22}$. Indeed, due to the reaction $\mathrm{B} \rightarrow 0$ (with rate constant $\left.k_{0}\right), \operatorname{det}(\mathbf{J})$ is positive for system (56); and the condition for Turing instability now reads

$$
\begin{aligned}
D_{21} k_{2} & +D_{12} k_{1}+D_{11}\left(k_{2}+k_{0}\right)+D_{22} k_{1} \\
& <-2[\operatorname{det}(\mathbf{D}) \operatorname{det}(\mathbf{J})]^{1 / 2}<0
\end{aligned}
$$

Comparing (57) to our earlier result (49) without the step B $\rightarrow 0$

$$
D_{21} k_{2}+D_{12} k_{1}+D_{11} k_{2}+D_{22} k_{1}<0
$$

we see that $D_{12}$ or $D_{21}$ for system (56) must be even more negative than for system (33) for Turing patters to occur. So, if Turing patterns can occur in system (56), then they can occur in the closed equilibrium system (33), which would contradict the second law of thermodynamics. Thus, the rate and diffusion coefficients cannot be arbitrarily chosen independently of one another; there must be some co-dependence. 
Tran-Cong and collaborators have found Turing patterns experimentally in a system that can be described by eqn (33), where $\mathrm{A}$ and $\mathrm{B}$ are polymers that can phase separate and can also undergo a reversible photoinduced interconversion. ${ }^{159,160,176-178}$ Phase separation in binary mixtures is related to cross-diffusion in that $\mathrm{A}$ molecules tend to diffuse toward $\mathrm{A}$ molecules and $\mathrm{B}$ toward $\mathrm{B}$ (clustering), and clusters $\mathrm{A}_{n}$ diffuse toward regions with lower concentrations of clusters $\mathrm{B}_{n}$ and vice versa. Though system (33) is not completely equivalent to a phase-separating system, we can expect Turing patterns in system (33) with appropriate crossdiffusion coefficients when the rate constant $k_{1}$ (or $k_{2}$ ) is a function of the light intensity. Note that the system (33) is now an open system with an influx of energy, so no contradiction of thermodynamics is implied.

Consider now what induces the autocatalytic growth of small perturbations in our linear system (33) if $D_{k}<0$ (cf. eqn (49)). For simplicity, suppose that $-D_{12}>D_{11} k_{2} / k_{1}+D_{22}$ (condition 49 with $D_{21}=0$ ). The result of a small perturbation of $b_{\text {ss }}$ at $x=x_{0}$ is plotted as curve " $b$ " in Fig. 5a. Due to the negative cross-diffusion coefficient $D_{12}$, a flux of species $a$, $D_{12} \partial b / \partial x$, is directed toward the center of perturbation (horizontal arrow in Fig. 5a) and creates there an excess of $a$ at the expense of $a$ in more remote areas (curve " $a$ "). Since $a$ increases in the center, further increase in $b$ due to the photoinduced reaction $\mathrm{A} \rightarrow \mathrm{B}$ (term $k_{1} a$ and vertical arrow in Fig. 5a) occurs. This is a positive feedback loop, which we might call crossspatio-chemical autocatalysis.

The conditions for Turing instability simply imply that the homogeneous steady state is unstable, but say nothing about the state that will eventually emerge. To stabilize an inhomogeneous (Turing) pattern, the system (50), (51) must have one or more nonlinear terms that terminate the exponential growth, $\exp \left(\operatorname{Re}(\lambda)_{\max } t\right)$, of the initial small perturbation, where $\operatorname{Re}(\lambda)$ has a positive maximum, $\operatorname{Re}(\lambda)_{\max }$, at wavenumber $k=k_{\max }$. Unlike classical Turing systems, however, our linear reaction system does not contain such chemical nonlinear terms as, for example, $-b^{2}$. If the cross-diffusion terms $\left(D_{12}\right.$ and $\left.D_{21}\right)$ were constants, then the maximum and minimum values of $a$ (or $b$ )
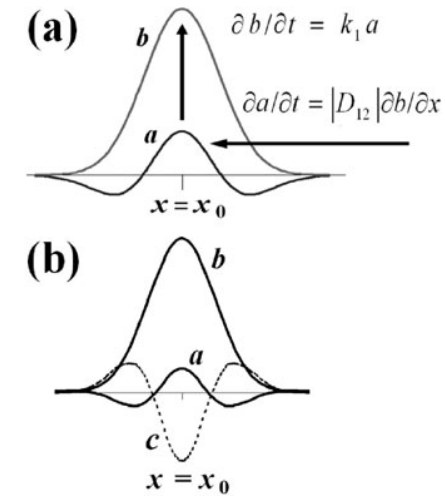

Fig. 5 (a) Schematic explanation of autocatalysis in linear reaction (33). (b) Profiles of species A (curve $a$ ), B (b), and C (c) after initial perturbation of $\mathrm{B}$ ( $\delta$-function at $x=x_{0}$ ) without any reaction between A, B, and C. Horizontal axis denotes homogeneous steady state levels for all species. Diffusion coefficients: $D_{12}=D_{21}=D_{31}=$ $D_{23}=0, D_{11}=1, D_{22}=0.3, D_{33}=0.2, D_{32}=0.2$, and $D_{13}=0.4$, time $t=0.3$. would approach $+\infty$ and $-\infty$, respectively. However, the dependences of $D_{12}$ and $D_{21}$ on $a$ and $b$, respectively, serve as a brake on the potentially infinite growth of $a$ and $b$, or, more precisely, these dependences prevent $a$ and $b$ from becoming negative. Indeed, at sufficiently small values of $a$ and $b, D_{12}$ and $D_{21}$ tend to zero, and the condition for Turing instability can no longer be satisfied. Since our system conserves mass, $\int_{\mathrm{v}} a+\int_{\mathrm{v}} b=$ constant (the subscript $\mathrm{V}$ signifies integration over the total volume of the system),i.e., the diffusive terms just redistribute concentrations but cannot change the total amount of matter, the limitation on concentration decrease implies a limitation on growth.

\section{B Nonreactive species}

Consider now the effect of nonreactive species in eqn (32). As the simplest case, we consider the photo-induced reaction (33) and add a nonreactive species $\mathrm{C}$. We know that a sufficiently large negative cross-diffusion coefficient $D_{12}$ can induce Turing instability in reaction (33). But suppose instead that $D_{12}=D_{21}=0$, i.e., there is no cross-diffusion in the reactive subsystem (A, B). Is it possible to mimic the needed coefficient $D_{12}$ by introducing crossfluxes between $\mathrm{A}$ and $\mathrm{C}$ and between $\mathrm{B}$ and $\mathrm{C}$ ? We answer this question in the affirmative by taking a hint from Fig. 5a. We seek cross-diffusion coefficients $D_{13}, D_{23}, D_{31}$, and $D_{32}$ (where the index 3 corresponds to C) to create a profile of species $a$ analogous to that in Fig. 5a. This task can be accomplished by using the analytical solution of the diffusion equations for three variables (equations and solutions analogous to eqn (35)-(43)). ${ }^{93}$ Avoiding these cumbersome equations, we first give a qualitative solution of the diffusion equations and then perform linear stability analysis and numerical solution of the corresponding RD system.

A negative coefficient $D_{12}$ would imply that a gradient in B creates a counter-flux of $\mathrm{A}$. Alternatively, if $\mathrm{B}$ produces a counter-flux of $\mathrm{C}$ (negative $D_{32}$ ) and in turn $\mathrm{C}$ generates a counter-flux of $\mathrm{A}$ (negative $D_{13}$ ), the net result should be equivalent to having a negative $D_{12}$. The same result is obtained if both $D_{32}$ and $D_{13}$ are positive. In Fig. 5 b we show this situation schematically for species $\mathrm{A}, \mathrm{B}$ and $\mathrm{C}$ without any reaction. Comparing the profiles of $a$ in Fig. 5a and b, we see that they are similar. Note that a positive coefficient $D_{i j}(i, j=1,2, i \neq j)$ can be replaced by a combination of positive and negative coefficients $D_{k j}$ and $D_{i k}$ ( $k=3$ for three variables); it does not matter which coefficient is chosen as positive and which as negative.

Consider now the same system more quantitatively by writing the following equations.

$$
\begin{aligned}
& \partial a / \partial t=-k_{1} a+k_{2} b+D_{11} \nabla^{2} a+\operatorname{div}\left(D_{\mathrm{AC}} \nabla c\right) \\
& \partial b / \partial t=k_{1} a-k_{2} b+D_{22} \nabla^{2} b+\operatorname{div}\left(D_{\mathrm{BC}} \nabla c\right) \\
& \partial c / \partial t=\operatorname{div}\left(D_{\mathrm{CA}} \nabla a\right)+\operatorname{div}\left(D_{\mathrm{CB}} \nabla b\right)+D_{33} \nabla^{2} c
\end{aligned}
$$

where we specify the concentration dependence of the crossdiffusion coefficients as $D_{\mathrm{AC}}=D_{13} a /\left(a+K_{\mathrm{A}}\right), D_{\mathrm{BC}}=$ $D_{23} b /\left(b+K_{\mathrm{B}}\right), D_{\mathrm{CB}}=D_{32} c /\left(c+K_{\mathrm{C}}\right), D_{\mathrm{CA}}=0$. In Fig. 6 we show the dispersion curves obtained from linear stability analysis of eqn (59)-(61) and the stationary Turing patterns found numerically in $1 \mathrm{D}$ for this system. The nonreactive 

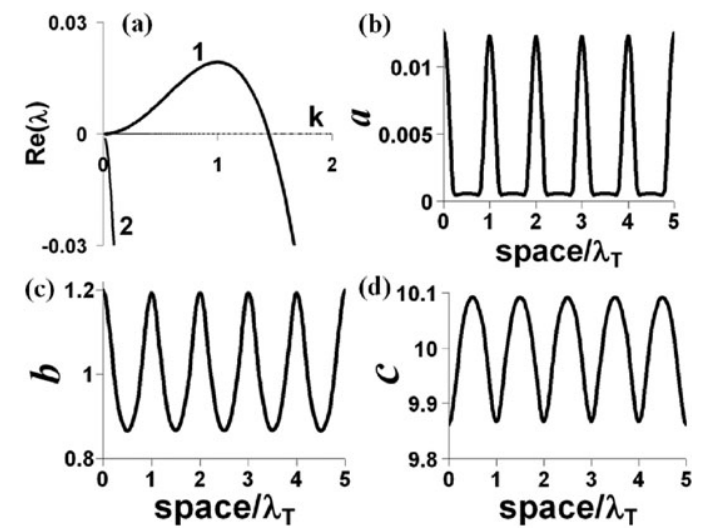

Fig. 6 Dispersion curves and Turing patterns in model eqn (59)-(61). Parameters: $k_{1}=30, k_{2}=0.1, D_{11}=2.5, D_{22}=2.5, D_{33}=1, D_{12}=$ $D_{21}=D_{31}=0, D_{13}=1.45, D_{23}=2.7, D_{32}=0.7, K_{\mathrm{A}}=0.0001$, $K_{\mathrm{B}}=0.1, K_{\mathrm{C}}=0.2$. Turing wavelength $\lambda_{\mathrm{T}}=6.3$. Curves 1 and 2 in (a) are different real eigenvalues.

variable $c$ (Fig. 6d) becomes spatially periodic like the reactive variables $a$ and $b$, but $180^{\circ}$ out of phase with them in analogy with the initial concentration distribution in Fig. 5b. The average values of all variables are equal to their initial steady state values. In this example we see that the nonreactive species $\mathrm{C}$ is an essential ingredient, since without $\mathrm{C}$ there are no patterns with $D_{12}=D_{21}=0$. In a system of this type, sprinkling $\mathrm{C}$ into a homogeneous mixture of $\mathrm{A}$ and $\mathrm{B}$ at equilibrium would result in the formation of Turing patterns.

A similar approach for pattern formation was used in a threecomponent phase-separating mixture of polymers, $\mathrm{A}+\mathrm{B}+\mathrm{C}$, where the component $\mathrm{C}$ is non-reactive. ${ }^{162,179,180}$ However, the notion of cross-diffusion was not explicitly invoked in these works.

\section{C Bimolecular reaction}

Thus far we have considered the role of cross-diffusion only in linear, monomolecular reactions. Consider now the more complex but more common case of bimolecular reactions of the general form

$$
\mathrm{A}+\mathrm{S} \leftrightarrow \mathrm{B}+\mathrm{P}
$$

with forward and reverse rate constants $k_{1}$ and $k_{2}$, respectively. As we did for reaction (33), we can seek the condition for Turing instability in this equilibrium reaction by applying linear stability analysis. To simplify our investigation and focus on the role of the cross-diffusion coefficients, we introduce just two nonzero cross-diffusion coefficients, $D_{12}$ and $D_{13}$ (where 1, 2, 3, 4 correspond to $\mathrm{A}, \mathrm{S}, \mathrm{B}$, and $\mathrm{P}$, respectively) between reacting species $\mathrm{A}$ and $\mathrm{S}$ and between reactant $\mathrm{A}$ and product $\mathrm{B}$, since the effect of the other cross-diffusion coefficients can be deduced from an understanding of the role played by these two coefficients.

If only $D_{12} \neq 0$ and all other off-diagonal elements of $\mathbf{D}$ are zero, then linear stability analysis gives the condition for Turing instability as

$$
\begin{aligned}
D_{12}> & D_{22}+D_{11}\left[\left(D_{22} D_{33} / D_{44}\right) k_{2} b+D_{22} k_{2} p\right. \\
& \left.+D_{33} k_{1} a\right] /\left(D_{33} k_{1} s\right)
\end{aligned}
$$

If only $D_{13} \neq 0$, then this condition takes the form

$$
\begin{aligned}
-D_{13}> & D_{33}+D_{11}\left[\left(D_{22} D_{33} / D_{44}\right) k_{2} b+D_{22} k_{2} p\right. \\
& \left.+D_{33} k_{1} a\right] /\left(D_{22} k_{1} s\right)
\end{aligned}
$$

The inequality (64) is similar to the condition (49) obtained for reaction (33) when $D_{21}=0$ :

$$
-D_{12}>D_{22}+D_{11} k_{2} / k_{1}
$$

Here $D_{13}$ (for reaction 62) is analogous to $D_{12}$ (for reaction 33), $D_{33}$ to $D_{22}, k_{2} b$ and $k_{2} p$ to $k_{2}$, and $k_{1} s$ and $k_{1} a$ to $k_{1}$.

To obtain Turing patterns in a system described by reaction (62), the coefficient $D_{13}$ must be negative or the coefficient $D_{12}$ positive. Intuitively, positive $D_{12}$ reduces the concentration of $\mathrm{S}$ at the site of a positive perturbation of $\mathrm{A}$ at $x=x_{0}$ (opposite of the situation shown in Fig. 5a). This decreases the rate of the forward reaction $\mathrm{A}+\mathrm{S}$ at $x=x_{0}$. As a result, $a$ locally increases due to the back reaction $\mathrm{B}+\mathrm{P}$, the initial positive perturbation $\delta a$ grows, and we have a positive feedback loop. Similarly, a negative $D_{13}$ implies that a positive perturbation of A draws B toward it, increasing the rate of the back reaction, thereby enhancing the initial perturbation of A. These cross-diffusion-induced effects must exceed the stabilizing effect of the diagonal diffusion terms if instability is to occur.

Again, if neither the forward or the reverse reaction in (62) is photoinduced, then conditions (63) and (64) are cannot hold from a thermodynamic point of view, since Turing patterns could then arise in a closed system. The inequalities (63) and (64) set constraints on the dependence of the mutual diffusion coefficients on the rate constants and equilibrium concentrations for bimolecular reactions. For example, from (63) it follows that at large $k_{1}$, the inequality $D_{12}<D_{22}+D_{11} a / s$ should hold, while at large $k_{2}$, we must have $D_{12}<D_{22}+$ $D_{11} D_{22}\left(k_{2} / k_{1}\right)\left(b / D_{44}+p / D_{33}\right) / s$.

If both the coefficients $D_{12}$ and $D_{13}$ for reaction (62) are nonzero, then Turing patterns can occur even if $D_{12}<D_{22}$ and $-D_{13}<D_{33}$. For example, at $k_{1}=10^{4} \mathrm{M}^{-1} \mathrm{~s}^{-1}$, $k_{2}=10^{3} \mathrm{M}^{-1} \mathrm{~s}^{-1}, a=10^{-5} \mathrm{M}, s=0.1 \mathrm{M}, b=10^{-4} \mathrm{M}$, and $p=0.1 \mathrm{M}$, a Turing instability exists when

$$
(\mathbf{D})=\left(\begin{array}{cccc}
1 & 0.52 & -0.51 & 0 \\
0 & 1 & 0 & 0 \\
0 & 0 & 1 & 0 \\
0 & 0 & 0 & 1
\end{array}\right)
$$

\section{D Complex systems (with autocatalysis)}

At the beginning of section 3, we mentioned several models, typically including autocatalytic reactions or their equivalent, in which introduction of cross-diffusion leads to pattern formation even with equal diagonal elements of the matrix D. ${ }^{132-142}$ Now we turn to more complex, and chemically realistic, activator-inhibitor systems.

We will look at several models of the well-known BelousovZhabotinsky (BZ) oscillatory reaction. ${ }^{181,182}$ Consider first a four-variable model, which is our reduction ${ }^{183}$ of the Field-Körös-Noyes (FKN) model. ${ }^{184}$ In the general eqn (32), the four dimensionless reactive species $x\left(1, \mathrm{HBrO}_{2}\right.$, activator), 
$y$ (2, $\mathrm{Br}^{-}$, inhibitor), $z$ (3, oxidized form of the catalyst), and $u\left(4, \mathrm{Br}_{2}\right)$ correspond to the following reactive terms:

$$
\begin{aligned}
& R_{1}=\left(1 / \varepsilon_{1}\right)\left(q y-x y+x-x^{2}\right) \\
& R_{2}=\left(1 / \varepsilon_{2}\right)\left[-2 q y-3 x y+f z-x^{2} / 2+\alpha u /(y+\gamma)\right] \\
& R_{3}=x-z \\
& R_{4}=\left(1 / \varepsilon_{4}\right)\left[q y+2 x y+x^{2} / 2-\alpha u /(y+\gamma)\right]
\end{aligned}
$$

There are no nonreactive species in this model. All the diagonal elements of the matrix $\mathbf{D}$ are taken to be 1 . We search for small cross-diffusion coefficients that strongly affect the stability of the homogeneous steady state [system (67)-(70) has only a single steady state]. Simulations of the BZ reaction typically employ two- or three-variable Oregonator models. ${ }^{185}$ We first explore here this four-variable model to demonstrate that other variables, like $\mathrm{Br}_{2}$ for example, which are usually ignored, can be critical for pattern formation. We find that the system behaviour is quite sensitive to all cross-diffusion coefficients $D_{i 4}$, while all coefficients $D_{4 j}$ have negligible effect. i.e., fluxes induced by gradients of bromine are important for pattern formation, while fluxes of bromine induced by gradients of other species are not. For example at the set of parameters $\left(f=1.2, q=1 \times 10^{-5}, \alpha=2 \times 10^{-5}, \gamma=0.2\right.$, $\left.\varepsilon_{1}=0.1, \varepsilon_{2}=0.004, \varepsilon_{4}=10^{-5}\right), D_{14}=-0.000025$ induces Turing instability, $D_{24}=-0.15$ induces wave instability, and $D_{34}=0.04$ induces both Turing and wave instability, while a negative $D_{34}\left(D_{34}=-0.001\right)$ induces only wave instability. If we eliminate the fourth variable $u$ from our model, we can seek to capture these effects by incorporating the effects of bromine cross-diffusion into the cross-diffusion coefficients of the remaining variables in the new reduced system. How to do this in a systematic fashion is an open question for the theory of RD systems.

If we are interested in the possibility of dissipative patterns resulting from cross-diffusion in an aqueous BZ solution, it is important to note that negative cross-diffusion coefficients $D_{24}$ between $\mathrm{Br}_{2}$ and $\mathrm{Br}^{-}$(due to complex, $\mathrm{Br}_{3}{ }^{-}$, formation) as well as between $\mathrm{Br}_{2}$ and the catalyst [Ru(bpy) ${ }_{3}$ or ferriin], $D_{34}$, can be relatively large at high $\mathrm{Br}^{-}$or catalyst concentrations, respectively, and at low temperatures.

Consider next the classical three-variable Oregonator model with the chemically reasonable reactions: ${ }^{185}$

$$
\begin{aligned}
\mathrm{Y} & \rightarrow \mathrm{X} \\
\mathrm{X}+\mathrm{Y} & \rightarrow 0 \\
\mathrm{X} & \rightarrow 2 \mathrm{X}+2 \mathrm{Z} \\
2 \mathrm{X} & \rightarrow 0 \\
\mathrm{Z} & \rightarrow h \mathrm{Y}
\end{aligned}
$$

After rescaling, the reactive terms $(1,2,3=\mathrm{X}, \mathrm{Y}, \mathrm{Z})$ are

$$
\begin{aligned}
& R_{1}=\left(1 / \varepsilon_{1}\right)\left(q y-x y+x-x^{2}\right) \\
& R_{2}=\left(1 / \varepsilon_{2}\right)(-q y-x y+f z) \\
& R_{3}=x-z
\end{aligned}
$$

where $f=2 h$. Again we consider a matrix $\mathbf{D}$ with diagonal elements $D_{i i}=1$ and search for cross-diffusion coefficients capable of destabilizing the homogeneous steady state. We find that three cross-diffusion coefficients are important for Turing instability, positive $D_{12}$ and $D_{31}$, and negative $D_{32}$ (we used $q=0.002, f=2.5, \varepsilon_{1}=0.05, \varepsilon_{2}=0.001$, but our conclusions hold for a broad range of other parameter sets). The coefficient $D_{12}$ can originate from reaction (71) or (72), where species X (1) and Y (2) both participate. $D_{31}$ arises from reaction (73), which involves $\mathrm{Z}$ and $\mathrm{X}$, and coefficient $D_{32}$ takes its importance from reaction (75).

Recall that positive (negative) cross-diffusion coefficients tend to separate (concentrate) the corresponding species. We know that $Y$ and $Z$ serve as inhibitors, while $X$ is the activator. We note that cross-diffusion processes that separate an activator and an inhibitor (positive $D_{12}$ and $D_{31}$ ) or draw together two inhibitors (negative $D_{32}$ ) appear to be important. Notice further that, like $D_{12}$ and $D_{31}$, positive cross-diffusion coefficients $D_{21}$ and $D_{13}$ also separate activator and inhibitor but have little effect; negative $D_{23}$ also has negligible effect on the stability of the steady state. The direction of the chemical reactions: (71) $2 \rightarrow 1$, (73) $1 \rightarrow 3$, and (75) $3 \rightarrow 2$ may explain the difference between the symmetrically related elements of $\mathbf{D}$; however, in complex nonlinear systems, such explanations may prove to be illusive.

\section{E Nonreactive species in complex systems}

We now explore briefly the introduction of nonreactive species, described in subsection 4B, to the Oregonator as an example of how addition of a fourth, nonreactive species (reactive term $R_{4}=0$ ) can mimic important cross-diffusion coefficients. To do this, we set all off-diagonal elements of $\mathbf{D}$ between reactive species to zero and again take all $D_{i i}=1$. To mimic positive $D_{12}$ and $D_{31}$ and negative $D_{32}$ we test the following pairs of crossdiffusion coefficients between reactive and nonreactive species: $D_{42}$ and $D_{14}$ of opposite sign for positive $D_{12}, D_{41}$ and $D_{34}$ of opposite sign for positive $D_{31}$, and $D_{42}$ and $D_{34}$ of the same sign for negative $D_{32}$. In all cases this strategy works quite well, independent of the choice of signs in any pair of cross-diffusion coefficients and even of the values of the individual coefficients. The onset of instability depends only on the product of coefficients:

$$
\begin{aligned}
& D_{42} D_{34} \cong D_{32}{ }^{\mathrm{c}} \\
& D_{42} D_{14} \cong D_{12}{ }^{\mathrm{c}} \\
& D_{41} D_{34} \cong D_{31}{ }^{\mathrm{c}}
\end{aligned}
$$

where $D_{32}{ }^{\mathrm{c}}, D_{12}{ }^{\mathrm{c}}$, and $D_{31}{ }^{\mathrm{c}}$ correspond to the onset of Turing instability in a system without component 4, i.e., system (76)-(78) with a single nonzero cross-diffusion coefficient.

We also find that if we change the sign of one of the cross-diffusion coefficients in the pairs considered above and slightly increase the magnitude of their product, we can obtain wave instability instead of Turing instability. For example, $D_{32}=-0.005$ gives Turing instability for system (76)-(78) (all other cross-diffusion coefficients are 0). At $D_{32}=0$ and $D_{42}=0.1, D_{34}=0.05$, we have Turing instability, while at $D_{42}=0.15, D_{34}=-0.2\left(\right.$ or $\left.D_{42}=-0.15, D_{34}=0.2\right)$, we have wave instability. 
If we add a second nonreactive species, more complex combinations of cross-diffusion coefficients can generate instability. Consider, as an example, the two-variable ( $x$ and $z$ ) Oregonator model $^{186}$

$$
\begin{aligned}
& R_{1}=(1 / \varepsilon)\left[x-x^{2}-f z(x-q) /(x+q)\right] \\
& R_{2}=x-z
\end{aligned}
$$

A positive cross-diffusion coefficient $D_{21}$, which separates activator $x$ and inhibitor $z$, can induce Turing instability at $D_{11}=D_{22}=1\left(q=0.002, f=2.5, \varepsilon_{1}=0.05, D_{21}{ }^{\mathrm{c}}=0.59\right.$, $D_{12}=0$ ). If we add one nonreactive species (3) and set $D_{21}$ to zero, then a combination of coefficients $D_{31}$ and $D_{23}$ with $D_{31} D_{23}=-0.59=-D_{21}{ }^{\text {c }}$ gives Turing instability. If we add the second nonreactive species, then a combination of $D_{31}$, $D_{43}$, and $D_{24}$ such that $D_{31} D_{43} D_{24}=0.59$ also gives Turing instability. Notice that the product of the three coefficients should be positive. In general, a combination of cross-diffusion coefficients between reactive $(i, j)$ and nonreactive $(k, m)$ species $D_{k j}, D_{m k}$, and $D_{i m}$ can replace the cross-diffusion coefficient $D_{i j}$ between two reactive species $i$ and $j$, if

$$
D_{k j} D_{m k} D_{i m}=D_{i j}
$$

This result can be extended to larger numbers of nonreactive species, though the likelihood of being able to realize such a system experimentally is small.

Wave instability also can be found in system (82)-(83) with additional nonreactive species $\left(q=0.001, f=2.3, \varepsilon_{1}=0.1\right.$, $D_{11}=D_{22}=1, D_{33}=1.3, D_{44}=3, D_{13}=-0.1, D_{34}=13$, $D_{42}=-60$, other off-diagonal elements of matrix $\mathbf{D}$ are zero). Notice that if we have only reactive species, then wave instability in systems like (2) or (3) requires at least three variables. If additional nonreactive species and cross-diffusion are present, two reactive species are sufficient.

Efforts are currently underway to find species that can be added to the BZ reaction to obtain Turing patterns, standing waves, or other spatiotemporal structures. Researchers have added various surfactants (at levels above the critical micelle concentration) or polymers. What is important to keep in mind in this search that such additives must interact with at least two different species in order to mimic cross-diffusion between them, for example, between the catalyst and $\mathrm{Br}_{2}$ or between $\mathrm{Br}^{-}$and $\mathrm{HBrO}_{2}$. If the additive interacts only with a single species, cross-diffusion cannot arise. Another strategy, which has not yet been implemented, would be to utilize two additives, each of which interacts with a different BZ species and in turn interact in an appropriate manner with each other, for example via excluded volume or electrostatic mechanisms.

\section{Conclusion}

To date, cross-diffusion has been almost completely ignored in efforts to explain dissipative patterns found in RD systems. Our theoretical analysis shows that this phenomenon has the potential to play an important role in pattern formation, even when the cross-diffusion coefficients are relatively small, if the kinetics are sufficiently nonlinear. At present we know three pattern-forming systems: the CDIMA and FIS reactions, and the BZ-AOT system, in which stationary patterns have been found, and of course, the $\mathrm{BZ}$ reaction itself, where waves are the norm. Recent experiments reveal that cross-diffusion coefficients in the BZ-AOT system are quite significant. ${ }^{93}$ Therefore cross-diffusion may be an important element in understanding the many types of patterns found in this system. The CDIMA $^{5,6}$ and the FIS $^{187}$ reactions probably merit further analysis, since cross-diffusion in these systems can result from electrostatic, excluded volume (diffusion in pores) and complexation effects. Such nonreactive species as starch or polyvinyl alcohol, which are typically added to the CDIMA reaction as indicators, can complex $\mathrm{I}_{3}{ }^{-}$and block gel pores to induce cross-diffusion.

We predict that dissipative patterns should occur in photoinduced linear (reversible) reactions with appropriate crossdiffusion coefficients or with additional nonreactive species (like polymers, charged particles or micelles) that can mimic cross-diffusion coefficients between reactive species. The wavelength of these patterns will depend strongly on the rate constants (which can be regulated by the light intensity) and should lie in the range from tens of nanometers to tens of micrometers. This may have practical application.

Further work is needed to elucidate the dependence of crossdiffusion coefficients on concentrations and, especially, on rate constants. As chemists become more aware of the capacity of cross-diffusion to generate patterns, and as they develop better intuition about how such processes occur, they are likely to be able to exploit cross-diffusion as a tool to design systems with desired properties rather than regarding it merely as an exotic phenomenon that cannot be found in textbook treatments of diffusion.

\section{Acknowledgements}

This work was supported by the National Science Foundation under CHE-0526866 and CHE-0615507. We thank Alexander Cherkashin, Ethan Meltzer and Federico Rossi for their experimental assistance and Raymond Kapral and Onofrio Annunziata for helpful discussions.

\section{References}

1 T. Yamaguchi, N. Suematsu and H. Mahara, in Nonlinear Dynamics in Polymer Systems, ed. J. Pojman and Q. TranCong-Miyata, ACS Symp. Ser. 869, 2004, pp. 16-27.

2 K. G. Libbrecht, The Snowflakes, 2003.

3 A. M. Turing, Philos. Trans. R. Soc. London, Ser. B, 1952, 237, $37-72$.

4 G. Nicolis and I. Prigogine, Self-Organization in Nonequilibrium Systems, Wiley-Interscience, New York, 1977.

5 V. Castets, E. Dulos, J. Boissonade and P. De Kepper, Phys. Rev. Lett., 1990, 64, 2953-2956.

6 Q. Ouyang and H. L. Swinney, Nature, 1991, 352, 610-612.

7 V. K. Vanag and I. R. Epstein, Phys. Rev. Lett., 2001, 87, 228301.

8 A. T. Winfree, Science, 1972, 175, 634-636.

9 V. K. Vanag and I. R. Epstein, Phys. Rev. Lett., 2002, 88, 088303.

10 M. Von Smoluchowski, Z. Phys. Chem., 1917, 92, 192.

11 R. M. Noyes, J. Am. Chem. Soc., 1956, 78, 5486-5490.

12 R. M. Noyes, Prog. React. Kinet. Mech., 1961, 1, 129-160.

13 M. Tachiya, Radiat. Phys. Chem., 1983, 21, 167-175.

14 V. K. Vanag and I. R. Epstein, Science, 2001, 294, 835-837.

15 K. J. Lee, W. D. McCormick, J. E. Pearson and H. L. Swinney, Nature, 1994, 369, 215-218. 
16 K. J. Lee and H. L. Swinney, Int. J. Bifurcation Chaos, Appl. Sci., 1997, 7, 1149-1158.

17 K. J. Lee, W. D. McCormick, Q. Ouyang and H. L. Swinney, Science, 1993, 261, 192-194.

18 A. Kaminaga, V. K. Vanag and I. R. Epstein, Angew. Chem., Int. Ed., 2006, 45, 3087-3089.

19 S. Koga and Y. Kuramoto, Prog. Theor. Phys., 1980, 63, 106-121.

20 J. E. Pearson, Science, 1993, 261, 189-192.

21 V. K. Vanag and I. R. Epstein, Chaos, 2007, 17, 037110.

22 R. Krishna and J. A. Wesselingh, Chem. Eng. Sci., 1997, 52, 861-911.

23 D. G. Leaist and F. F. Al-Dhaher, J. Chem. Eng. Data, 2000, 45, 308-314.

24 J. W. Fu, L. Paduano, J. A. Rard, J. G. Albright and D. G. Miller, J. Chem. Eng. Data, 2001, 46, 601-608.

25 J. W. Fu, J. A. Rard, L. Paduano, J. G. Albright and D. G. Miller, J. Chem. Eng. Data, 2002, 47, 496-512.

26 D. G. Leaist and S. M. Abdu, J. Chem. Eng. Data, 2001, 46, 922-926.

27 M. Thies, S. F. Clancy and H. H. Paradies, J. Phys. Chem., 1996, 100, 9881-9891.

28 D. G. Leaist and L. Hao, J. Phys. Chem., 1995, 99, 12896-12901.

29 O. Annunziata, L. Paduano and J. G. Albright, J. Phys. Chem. B, 2006, 110, 16139-16147.

30 A. Vergara, L. Paduano and R. Sartorio, Phys. Chem. Chem. Phys., 2001, 3, 4340-4345.

31 A. Vergara, L. Paduano and R. Sartorio, Macromolecules, 2002, 35, 1389-1398.

32 E. O. Budrene and H. C. Berg, Nature, 1995, 376, 49-53.

33 J. D. Murray, Mathematical Biology. II, Spatial Models and Biomedical Applications, Springer, NY, 3rd. edn., 2003.

34 B. N. Vasiev, P. Hogeweg and A. V. Panfilov, Phys. Rev. Lett., 1994, 73, 3173-3176.

35 M. A. Tsyganov, J. Brindley, A. V. Holden and V. N. Biktashev, Physica D, 2004, 197, 18-33.

36 M. A. Tsyganov, V. N. Biktashev, J. Brindley, A. V. Holden and G. R. Ivanitsky, Physics-Uspekhi, 2007, 50, 263-286.

37 M. X. Wang, Comput. Math. Appl., 2006, 52, 707-720.

38 X. Z. Zeng, J. Math. Anal. Appl., 2007, 332, 989-1009.

39 W. W. Tso and J. Adler, J. Bacteriol., 1974, 118, 560-576.

40 R. Mathew, L. Paduano, J. G. Albright, D. G. Miller and J. A. Rard, J. Phys. Chem., 1989, 93, 4370-4374.

41 L. Paduano, R. Sartorio, V. Vitagliano, J. G. Albright, D. G. Miller and J. Mitchell, J. Phys. Chem., 1990, 94, 6885-6888.

42 F. Capuano, A. Vergara, L. Paduano, O. Annunziata and R. Sartorio, J. Phys. Chem. B, 2003, 107, 12363-12369.

43 H. C. Halvorsen and D. G. Leaist, Phys. Chem. Chem. Phys., 2004, 6, 3515-3523.

44 J. G. Albright, L. Paduano, R. Sartorio, A. Vergara and V. Vitagliano, J. Chem. Eng. Data, 2001, 46, 1283-1291.

45 A. Vergara, L. Paduano, V. Vitagliano and R. Sartorio, J. Phys. Chem. B, 2000, 104, 8068-8074.

46 A. Vergara, L. Paduano and R. Sartorio, J. Phys. Chem. B, 2001, 105, 328-334.

47 A. Vergara, L. Paduano, V. Vitagliano and R. Sartorio, Macromolecules, 2001, 34, 991-1000.

48 A. Vergara, O. Annunziata, L. Paduano, D. G. Miller, J. G. Albright and R. Sartorio, J. Phys. Chem. B, 2004, 108, 2764-2772.

49 A. Vergara, F. Capuano, L. Paduano and R. Sartorio, Macromolecules, 2006, 39, 4500-4506.

50 H. L. Toor, AIChE J., 1957, 3, 198-207.

51 S. Emmanuel, A. Cortis and B. Berkowitz, Chem. Phys., 2004, 302, 21-30.

52 K. MacEwan and D. G. Leaist, J. Phys. Chem. B, 2002, 106, 10296-10300.

53 D. G. Miller, V. Vitagliano and R. Sartorio, J. Phys. Chem., 1986, 90, 1509-1519.

54 T. Graham, Trans. R. Soc. London, 1850, 140, 1.

55 A. E. Fick, Ann. Phys. (Poggendorf), 1855, 94, 59.

56 L. Onsager and R. M. Fuoss, J. Phys. Chem., 1932, 36, 2689-2778.

57 R. L. Baldwin, P. J. Dunlop and L. J. Gosting, J. Am. Chem. Soc., $1955,77,5235-5238$.
58 P. J. Dunlop and L. J. Gosting, J. Am. Chem. Soc., 1955, 77, $5238-5249$.

59 E. L. Cussler and P. J. Dunlop, J. Phys. Chem., 1966, 70, $1880-1888$.

60 O. Annunziata, A. Vergara, L. Paduano, R. Sartorio, D. G. Miller and J. G. Albright, J. Phys. Chem. B, 2003, 107, 6590-6597.

61 D. G. Leaist, J. Chem. Soc., Faraday Trans. 1, 1987, 83, 829-839.

62 R. A. Noulty and D. G. Leaist, J. Phys. Chem., 1987, 91, $1655-1658$.

63 G. P. Rai and H. T. Cullinan, J. Chem. Eng. Data, 1973, 18, 213-214.

64 G. I. Taylor, Proc. R. Soc. Lond. A, 1953, 219, 186-203.

65 W. E. Price, J. Chem. Soc., Faraday Trans. 1, 1988, 84, 2431-2439.

66 A. Alizadeh, C. A. N. DeCastrro and W. A. Wakeham, Int. J. Thermophys., 1980, 1, 243-284.

67 R. Aris, Proc. R. Soc. Lond. A, 1956, 235, 67-77.

68 R. Mills, J. Phys. Chem., 1965, 69, 3116-3119.

69 J. G. Albright and R. Mills, J. Phys. Chem., 1965, 69, 3120-3126.

70 H. J. V. Tyrrell and K. R. Harris, Diffusion in Liquids: A Theoretical and Experimental Study, Butterworths, London, 1984.

71 J. P. Hansen and I. R. McDonald, Theory of Simple Liquids, Academic Press, New York, 1986.

72 H. G. Hertz, Ber. Bunsen-Ges., 1977, 81, 656-664.

73 Y. H. Zhou and G. H. Miller, J. Phys. Chem., 1996, 100, $5516-5524$.

74 A. C. Lasaga, Geochim. Cosmochim. Acta, 1979, 43, 455-469.

75 L. S. Darken, Trans. AIME, 1948, 175, 184-201.

76 D. C. Douglass and H. L. Frisch, J. Phys. Chem., 1969, 73, 3039-3047.

77 R. J. Bearman, J. Phys. Chem., 1961, 65, 1961.

78 D. W. McCall and D. C. Douglass, J. Phys. Chem., 1967, 71, 987.

79 S. R. DeGroot and P. Mazur, Non-Equilibrium Thermodynamics, Dover, New York, 1984.

80 H. T. Cullinan, Industrial \& Engineering Chemistry Fundamentals, 1965, 4, 133.

81 J. S. Kirkaldy, D. Weichert and Zia-Ul-Haq, Can. J. Phys., 1963, 41, 2166-2173.

82 W. E. Stewart and R. Prober, Industrial \& Engineering Chemistry Fundamentals, 1964, 3, 224.

83 H. L. Toor, AIChE J., 1964, 10, 448-455.

84 H. L. Toor, AIChE J., 1964, 10, 460-465.

85 H. L. Toor, AIChE J., 1964, 10, 545-548.

86 D. Kondepudi and I. Prigogine, Modern Thermodynamics, John Wiley \& Sons, Chichester, UK, 1998.

87 O. Annunziata, L. Paduano, A. J. Pearlstein, D. G. Miller and J. G. Albright, J. Am. Chem. Soc., 2000, 122, 5916-5928.

88 E. P. Zemskov, K. Kassner and M. J. B. Hauser, Phys. Rev. E, 2008, 77, 036219.

89 M. A. Tsyganov, J. Brindley, A. V. Holden and V. N. Biktashev, Phys. Rev. Lett., 2003, 91, 218102.

90 D. A. Ivanov, T. Grossmann and J. Winkelmann, Fluid Phase Equilib., 2005, 228, 283-291.

91 K. MacEwan and D. G. Leaist, Phys. Chem. Chem. Phys., 2003, 5, 3951-3958.

92 D. G. Leaist, Ber. Bunsen-Ges. Phys. Chem., 1991, 95, 117-122.

93 V. K. Vanag, F. Rossi, A. A. Cherkashin and I. R. Epstein, J. Phys. Chem. B, 2008, 112, 9058-9070.

94 A. Bardow, V. Goke, H. J. Koss and W. Marquardt, AIChE J., 2006, 52, 4004-4015.

95 G. S. Hartley, Philos. Mag., 1931, 12, 473.

96 L. J. Gosting, Adv. Protein Chem., 1956, 11, 429-554.

97 J. Barthel, H. Krienke and W. Kunz, Physical Chemistry of Electrolyte Solutions, Steinkopff, Darmstadt, 1998.

98 H. Treml and H. H. Kohler, Chem. Phys., 2000, 252, 199-208.

99 J. Barthel, H. J. Gores, C. M. Lohr and J. J. Seidl, J. Solution Chem., 1996, 25, 921-935.

100 V. Vitagliano and R. Sartorio, J. Phys. Chem., 1970, 74, 2949-2956.

101 D. G. Leaist, J. Phys. Chem., 1988, 92, 5833-5836.

102 D. G. Leaist, Can. J. Chem., 1988, 66, 1129-1134.

103 H. Kim, J. Solution Chem., 1974, 3, 271-287.

104 D. Leaist, J. Chem. Soc., Faraday Trans. 1, 1982, 78, 3069-3079. 
105 L. J. Gosting, H. Kim, M. A. Loewenst, G. Reinfeld and A. Revzin, Rev. Sci. Instrum., 1973, 44, 1602-1609.

106 J. A. Rard, J. G. Albright, D. G. Miller and M. E. Zeidler, J. Chem. Soc., Faraday Trans., 1996, 92, 4187-4197.

107 J. G. Albright, R. Mathew, D. G. Miller and J. A. Rard, J. Phys. Chem., 1989, 93, 2176-2180.

108 R. Mathew, J. G. Albright, D. G. Miller and J. A. Rard, J. Phys. Chem., 1990, 94, 6875-6878.

109 D. G. Miller, J. G. Albright, R. Mathew, C. M. Lee, J. A. Rard and L. B. Eppstein, J. Phys. Chem., 1993, 97, 3885-3899.

110 C. Tanford, Physical Chemistry of Macromolecules, Wiley, New York, 1961.

111 D. G. Leaist and L. Hao, J. Chem. Soc., Faraday Trans., 1993, 89, 2775-2782.

112 C. Tanford, S. A. Swanson and W. S. Shore, J. Am. Chem. Soc., 1955, 77, 6414-6421.

113 J. S. Newman, Electrochemical Systems, Prentice Hall, Englewood Cliffs, 1973.

114 D. G. Leaist and L. Hao, J. Phys. Chem., 1993, 97, 7763-7768.

115 P. Roscigno, L. Paduano, G. D'Errico and V. Vitagliano, Langmuir, 2001, 17, 4510-4518.

116 D. G. Leaist, Phys. Chem. Chem. Phys., 2002, 4, 4732-4739.

117 L. Costantino, C. Dellavolpe, O. Ortona and V. Vitagliano, J. Chem. Soc., Faraday Trans., 1992, 88, 61-63.

118 M. Bär, S. Nettesheim, H. H. Rotermund, M. Eiswirth and G. Ertl, Phys. Rev. Lett., 1995, 74, 1246-1249.

119 M. Bär, M. Eiswirth, H. H. Rotermund and G. Ertl, Phys. Rev. Lett., 1992, 69, 945-948.

120 G. Ertl, Science, 1991, 254, 1750-1755.

121 G. Ertl, Faraday Discuss., 2002, 121, 1-15.

122 M. Hildebrand, M. Ipsen, A. S. Mikhailov and G. Ertl, New J. Phys., 2003, 5, 61.

123 A. S. Mikhailov and G. Ertl, Chaos, 2002, 12, 107.

124 A. von Oertzen, H. H. Rotermund, A. S. Mikhailov and G. Ertl, J. Phys. Chem. B, 2000, 104, 3155-3178.

125 S. Sasaki and H. Maeda, Bull. Chem. Soc. Jpn., 1996, 69, 2173-2178.

126 O. Rosen, M. Bostrom, M. Nyden and L. Piculell, J. Phys. Chem. $B, 2003,107,4074-4079$.

127 F. Tsunomori and H. Ushiki, Phys. Lett. A, 1999, 258, 171-176.

128 E. N. Dunmire, A. M. Plenys and D. F. Katz, J. Controlled Release, 1999, 57, 127-140.

129 J. Tong and J. L. Anderson, Biophys. J., 1996, 70, 1505-1513.

130 R. S. Shaw, N. Packard, M. Schroter and H. L. Swinney, Proc. Natl. Acad. Sci. U. S. A., 2007, 104, 9580-9584.

131 R. Pei, S. K. Taylor, D. Stefanovic, S. Rudchenko, T. E. Mitchell and M. N. Stojanovic, J. Am. Chem. Soc., 2006, 128, 12693-12699.

132 C. J. Roussel and M. R. Roussel, Prog. Biophys. Mol. Biol., 2004, 86, $113-160$

133 M. R. Roussel and J. Wang, Phys. Rev. Lett., 2001, 87, 188302.

134 H. Malchow, J. Theor. Biol., 1988, 135, 371-381.

135 D. del-Castillo-Negrete, B. A. Carreras and V. Lynch, Physica D, 2002, 168, 45-60.

136 S. Kovacs, Nonlinear Analysis-Theory Methods \& Applications, 2004, 59, pp. 567-581.

137 H. A. Abdusalam and E. S. Fahmy, Chaos, Solitons Fractals, 2003, 18, 259-266.

138 J. E. Satulovsky, J. Theor. Biol., 1996, 183, 381-389.

139 P. K. Tapaswi and J. Chattopadhyay, Biosystems, 1993, 29, 25-36.

140 V. A. Vasilev, Ju. M. Romanovsky and V. G. Yakhno, Autowave Processes, ed. D. S. Chernavsky, Nauka, Moscow, 1987.

141 J. M. Chung and E. Peacock-López, J. Chem. Phys., 2007, 127, 174903

142 J. M. Chung and E. Peacock-López, Phys. Lett. A, 2007, 371, 41-47.

143 Y. Almirantis and S. Papageorgiou, J. Theor. Biol., 1991, 151, 289-311.

144 J. Adler and W. W. Tso, Science, 1974, 184, 1293-1294.

145 D. Lauffenburger, R. Aris and K. Keller, Biophys. J., 1982, 40, 209-219.

146 L. Dung, Appl. Math. Comput., 2002, 131, 531-558.
147 M. Shachak, B. Boeken, E. Groner, R. Kadmon, Y. Lubin, E. Meron, G. Ne'Eman, A. Perevolotsky, Y. Shkedy and E. D. Ungar, Bioscience, 2008, 58, 209-221.

148 E. Gilad, J. von Hardenberg, A. Provenzale, M. Shachak and E. Meron, J. Theor. Biol., 2007, 244, 680-691.

149 E. Meron, E. Gilad, J. von Hardenberg, M. Shachak and Y. Zarmi, Chaos, Solitons Fractals, 2004, 19, 367-376.

150 J. von Hardenberg, E. Meron, M. Shachak and Y. Zarmi, Phys. Rev. Lett., 2001, 8719, 198101.

151 A. J. Lotka, J. Am. Chem. Soc., 1920, 42, 1595-1599.

152 D. del-Castillo-Negrete and B. A. Carreras, Phys. Plasmas, 2002, 9, 118-127.

153 J. M. Epstein, Nonlinear Dynamics, Mathematical Biology, and Social Science, Addison-Wesley, Reading, MA, 1997, pp. 97-99.

154 M. Bahiana and Y. Oono, Phys. Rev. A, 1990, 41, 6763-6771.

155 S. C. Glotzer, E. A. Dimarzio and M. Muthukumar, Phys. Rev. Lett., 1995, 74, 2034-2037.

156 Y. Oono and M. Bahiana, Phys. Rev. Lett., 1988, 61, 1109-1111.

157 Y. Oono and M. Bahiana, J. Phys.: Condens. Matter, 1989, 1, $5297-5299$.

158 Q. Tran-Cong-Miyata, S. Nishigami, T. Ito, S. Komatsu and T. Norisuye, Nat. Mater., 2004, 3, 448-451.

159 Q. Tran-Cong, J. Kawai and K. Endoh, Chaos, 1999, 9, 298-307.

160 Q. Tran-Cong and A. Harada, Phys. Rev. Lett., 1996, 76, 1162-1165.

161 R. D. M. Travasso, O. Kuksenok and A. C. Balazs, Langmuir, 2005, 21, 10912-10915.

162 R. D. M. Travasso, O. Kuksenok and A. C. Balazs, Langmuir, 2006, 22, 2620-2628.

163 Y. De Decker, H. Marbach, M. Hinz, S. Gunther, M. Kiskinova, A. S. Mikhailov and R. Imbihl, Phys. Rev. Lett., 2004, 92, 198305.

164 Y. De Decker and A. S. Mikhailov, Prog. Theor. Phys. Suppl., 2006, 165, 119-143.

165 M. Hinz, S. Gunther, H. Marbach and R. Imbihl, J. Phys. Chem. $B, 2004,108,14620-14626$.

166 H. Marbach, S. Gunther, B. Luerssen, L. Gregoratti, M. Kiskinova and R. Imbihl, Catal. Lett., 2002, 83, 161-164.

167 H. Marbach, M. Hinz, S. Gunther, L. Gregoratti, M. Kishkinova and R. Imbihl, Chem. Phys. Lett., 2002, 364, 207-212.

168 H. Marbach, G. Lilienkamp, H. Wei, S. Gunther, Y. Suchorski and R. Imbihl, Phys. Chem. Chem. Phys., 2003, 5, 2730-2735.

$169 \mathrm{Y}$. Xu, H. Marbach, R. Imbihl, I. G. Kevrekidis and M. Mavrikakis, J. Phys. Chem. C, 2007, 111, 7446-7455.

170 I. R. Epstein and V. K. Vanag, Chaos, 2005, 15, 047510.

171 V. K. Vanag, Phys.-Usp., 2004, 47, 923-941.

172 V. K. Vanag and I. R. Epstein, in Self-Organized Morphology in Nanostructured Materials, ed. K. Al-Shamery and J. Parisi, Springer, Berlin, 2008, pp. 89-113.

173 R. Lefever, D. Carati and N. Hassani, Phys. Rev. Lett., 1995, 75, 1674.

174 L. Matyas and P. Gaspard, Phys. Rev. E, 2005, 71, 036147.

175 I. R. Epstein and J. A. Pojman, An Introduction to Nonlinear Chemical Dynamics, Oxford University Press, New York, 1998.

176 H. Nakanishi, M. Satoh and Q. Tran-Cong-Miyata, Phys. Rev. E, 2008, 77, 020801.

177 A. Masunaga, S. Ishino, H. Nakanishi and Q. Tran-CongMiyata, Kobunshi Ronbunshu, 2007, 64, 294-300.

178 S. Ishino, H. Nakanishi, T. Norisuye, Q. Tran-Cong-Miyata and Y. Awatsuji, Macromol. Rapid Commun., 2006, 27, 758-762.

179 P. Dayal, O. Kuksenok and A. C. Balazs, Langmuir, 2008, 24, 1621-1624.

180 O. Kuksenok, R. D. M. Travasso and A. C. Balazs, Phys. Rev. E, 2006, 74, 011503.

181 B. P. Belousov, in Collection of Short Papers on Radiation Medicine, Medgiz, Moscow, 1959, pp. 145-152.

182 A. M. Zhabotinsky, Proc. Acad. Sci. U. S. S. R., 1964, 157, 392-395.

183 V. K. Vanag and I. R. Epstein, Phys. Rev. Lett., 2004, 92, 28301.

184 R. J. Field, E. Körös and R. M. Noyes, J. Am. Chem. Soc., 1972, 94, 8649-8664.

185 R. J. Field and R. M. Noyes, J. Chem. Phys., 1974, 60, $1877-1884$

186 J. P. Keener and J. J. Tyson, Physica D, 1986, 21, 307-324.

187 I. Szálai and P. De Kepper, Chaos, 2008, 18, 026105. 